1 Running title: Hippocampal inhibitory circuits underlying memory consolidation

\title{
A dentate gyrus-CA3 inhibitory circuit promotes evolution of hippocampal-cortical ensembles during memory consolidation
}

5 1,2,3 Hannah Twarkowski, 1,2,3 Victor Steininger, 1,2,3 Min Jae Kim, and 1,2,3,4,5 Amar Sahay

$6 \quad{ }^{1}$ Center for Regenerative Medicine, Massachusetts General Hospital, Boston, MA 02114, USA. ${ }^{2}$ Harvard Stem

7 Cell Institute, Cambridge, MA 02138, USA. ${ }^{3}$ Department of Psychiatry, Massachusetts General Hospital, Harvard

8 Medical School, Boston, MA 02114 USA. ${ }^{4}$ BROAD Institute of Harvard and MIT, Cambridge, MA 02142.

${ }^{5}$ Correspondence: asahay@mgh.harvard.edu

Abstract (150 words)

3 Memories encoded in the dentate gyrus (DG)-CA3 circuit of the hippocampus are routed from CA1 to anterior cingulate cortex (ACC) for consolidation. Although CA1 parvalbumin inhibitory neurons (PV INs) orchestrate hippocampal-cortical communication, we know less about CA3 PV INs or DG-CA3 principal neuron-IN circuit mechanisms that contribute to evolution of hippocampal-cortical ensembles during memory consolidation. Using viral genetics to selectively enhance dentate granule cell recruitment of CA3 PV INs and feed-forward inhibition (FFI) in CA3 and longitudinal in vivo calcium imaging, we demonstrate that FFI facilitates formation and maintenance of context-associated neuronal ensembles in CA1. Increasing FFI in DG-CA3 promoted context specificity of neuronal ensembles in ACC over time and enhanced long-term contextual fear memory. Our findings illuminate how FFI in DG-CA3 dictates evolution of ensemble properties in CA1 and ACC during memory consolidation and suggest a teacher-like function for hippocampal CA1 in stabilization and re3 organization of cortical representations. 


\section{Introduction}

The hippocampus plays a critical role in the formation of new episodic memories by generating conjunctive representations of experiences and transferring these representations to neocortical sites for memory storage or consolidation (Frankland \& Bontempi, 2005; McClelland et al., 1995; Peyrache et al., 2009; Squire, 2004; Wilson $\&$ McNaughton, 1994). Highly processed sensory information underlying experiences are encoded in the dentate gyrus (DG)-CA3 circuit as distinct or updated representations of prior experiences and subsequently, routed out of CA1 to prefrontal cortical sites such as the anterior cingulate cortex (ACC) for consolidation. Following learning, prefrontal cortical neuronal ensembles are thought to undergo time-dependent refinement and reorganization as memories transition from recent to remote configurations and become more generalized (DeNardo et al., 2019; Frankland \& Bontempi, 2005; Frankland et al., 2004; Maviel et al., 2004; McClelland et al., 1995; Morrissey et al., 2017; Takehara-Nishiuchi \& McNaughton, 2008; Wiltgen et al., 2010; Winocur et al., 2010). The hippocampus is theorized to play a continuous, potentially instructive role in transformation of memories from conjunctive, context-rich representations to semantic, gist-like structures (Goode et al., 2020; Nadel \& Moscovitch, 1997; Teyler \& DiScenna, 1986; Tonegawa et al., 2018; Winocur et al., 2010; Winocur et al., 2007). Indeed, a growing body of experimental evidence not only supports a sustained role for the hippocampus in remote memory retrieval (Goshen et al., 2011; Vetere et al., 2021) but also in dictating the extent to which remote memories retain contextual details of the original experience (Guo et al., 2018; Koolschijn et al., 2019a; Tompary \& Davachi, 2017).

Investigations into neural circuit mechanisms mediating memory consolidation has identified key roles for principal cells and inhibitory neurons in mediating hippocampal-cortical communication. Specifically, genetic silencing of engram-bearing dentate granule neurons post training was shown to impair the maturation of context-specific neuronal ensembles in prefrontal cortex (Kitamura et al., 2017). Chronic genetic inhibition of CA3 outputs impaired high-frequency network oscillations such as sharp-wave ripples, experience-related firing patterns in CA1 and long-term contextual fear memory (Nakashiba et al., 2009). Parvalbumin inhibitory neurons (PV INs) in CA1 are the most extensively studied with regards to their roles in memory consolidation. CA1 PV INs have been shown to orchestrate synchronous activation of principal neurons, promote sharp-wave ripples (SWR) and facilitate hippocampal-cortical communication through increased sharp-wave ripple-spindle coupling in CA1-ACC networks (Buzsaki, 2015; Caliskan et al., 2016; Gan et al., 2016; Ognjanovski et al., 2017; Stark et al., 2014; Xia et al., 2017). Chemogenetic inhibition of PV INs in CA1 or in ACC following training inhibited consolidation of contextual fear memory (Ognjanovski et al., 2017; Xia et al., 2017). Together, these observations begin to highlight the contribution of principal neurons and INs in CA1 and ACC in memory consolidation. However, we know significantly much less about the contributions of principal neurons-IN circuits in DG-CA3, let alone how PV INs in CA3 govern the evolution of neuronal ensembles in downstream CA1-ACC networks over time.

Dentate granule cell recruitment of feed-forward inhibition (FFI) onto CA3 following learning is one inhibitory circuit mechanism that is a substrate for memory consolidation (Guo et al., 2018; Ruediger et al., 2012; Ruediger et al., 2011). In previous work, we found that selectively enhancing dentate granule cell recruitment of PV INs in CA3 increased inhibition onto CA3 and enhanced strength and precision of remote memories (Guo et increasing FFI in DG-CA3 conferred context-specific reactivation of neuronal ensembles in hippocampal-ACC- 
a link between maintenance of the hippocampal memory trace and remote memory precision(Guo et al., 2018).

As with new findings, we were motivated to investigate how FFI in DG-CA3 impacts ongoing neuronal activity, maintenance and specificity of neuronal ensembles in hippocampal-cortical networks during memory consolidation. In this study, we sought to address this goal by combining viral genetics to selectively enhance FFI in DG-CA3 and longitudinal in vivo 1-P calcium imaging to record ensemble properties in CA1 and ACC over time. We found that enhancing FFI facilitates the formation and maintenance of context-associated neuronal ensembles in CA1. Stable and precise CA1 ensembles, in turn, promoted acquisition of context-specific neuronal ensembles in $\mathrm{ACC}$ at remote timepoint and enhanced long-term contextual fear memory. Our findings illuminate how FFI in DG-CA3 dictates evolution of ensemble properties in CA1 and ACC during memory consolidation and suggest a teacher-like function for hippocampal CA1 in stabilization and re-organization of cortical representations.

\section{Results}

\section{CA1-ACC cross region comparisons in learning-naïve mice following enhancement of FFI in DG-CA3}

To begin to understand how FFI in DG-CA3 contributes to evolution of ensemble properties during memory consolidation, we leveraged our recent discovery of a learning-regulated molecular brake of dentate granule cell connectivity with stratum lucidum PV INs in CA3. Viral downregulation of the cytoskeletal protein, Ablim3, in the DG (using validated shRNA vs. non-target scrambled NT) resulted in increased dentate granule cell mossy fiber contacts with PV INs and an increase in PV IN puncta or contacts with CA3. Consistently, ex vivo electrophysiological recordings showed that these anatomical changes in FFI connectivity resulted in increased feed-forward inhibition onto CA3 (Guo et al., 2018) (schematized in Figure 1a). Here, we delivered lentiviruses shRNA (shRNA) or non-target shRNA (shNT) into the DG in combination with viral expression of the genetically encoded calcium indicator GCaMP6f in CA1 or ACC (Figure 1b). We injected mice with lenti-shNT or lentishRNA in the DG and quantified PV puncta, an anatomical marker for PV mediated inhibition, in CA3, CA1 and ACC two weeks past injection. As previously shown (Guo et al., 2018), we found an increase in PV puncta in CA3 in shRNA injected mice [Two-tailed Mann-Whitney: $p=0.029, n=4$ ]. Consistent with prior reports (Soltesz \& Losonczy, 2018), we found a significantly higher number of PV puncta in the deeper layer of CA1 compared to the superficial layer [Two-way repeated measures ANOVA, layer x virus effect: N.S.: main virus effect N.S., main layer effect $\mathrm{p}<0.0001, \mathrm{n}=4$ ]. Our manipulation did not affect PV puncta in CA1 or ACC [ACC: Two-tailed Mann-Whitney test: N.S., n=4] (Figure 1c). Together, these data demonstrate that our viral manipulation increases FFI onto CA3 without affecting PV IN-principal neuron anatomical contacts in CA1 or ACC.

To investigate how enhancing FFI in DG-CA3 influences CA1 and ACC ensemble activity in learningnaïve mice and during acquisition and recall of recent and remote memories, we performed imaging in a cohort of mice in a contextual fear conditioning paradigm as schematized in Figure 1d. Two weeks following bilateral viral injections in DG (lenti-shNT or control mice vs. shRNA or experimental mice) and unilateral injections into CA1 or ACC (AAV1.CamKII.GCaMP6f), we recorded neuronal activity (calcium transients) using miniaturized endoscopes in CA1 or ACC. We performed recordings at same day baseline (BL), during contextual fear conditioning in the training context, context $\mathrm{A}$, and during recall in context $\mathrm{A}$ and a distinct, neutral context (context $\mathrm{C}$ ) at recent (day 1) and remote timepoints (day 16). To determine if enhancing FFI in DG-CA3 affects neuronal activity in CA1 and ACC in learning-naïve mice, we performed recordings during exploration of a familiar environment (day -3) (Figure 1e-i). We detected no virus-mediated effect when comparing within brain regions (data not shown). However, when comparing across regions, we detected significantly higher rate of 
calcium transients in CA1 compared to ACC neurons in lenti-shRNA mice [Two-way repeated measures ANOVA, brain region $x$ time effect $\mathrm{p}<0.0001$; brain region main effect $\mathrm{p}=0.046$, time main effect N.S., ACC $\mathrm{n}=5$, CA1 $\mathrm{n}=6$ ] but not in lenti-shNT mice [Two-way repeated measures ANOVA, brain region $\mathrm{x}$ time effect $\mathrm{p}<0.0001$; brain region main effect N.S., time main effect $\mathrm{p}=0.026, \mathrm{ACC} n=5, \mathrm{CA} 1 \mathrm{n}=5]$ (Figure 1f, g). Analysis of synchronized activity revealed that CA1 neurons were less co-active than ACC neurons in the experimental group (Figure 1h) [unpaired t-test with Welch's correction, shNT: ACC vs CA1 N.S., n=5; shRNA: ACC vs CA1: $p=0.0212, n=5$ ACC, $n=6$ CA1. These differences in neuronal activity were unlikely to be due to differences in locomotion (Kemere et al., 2013) since both control and experimental groups exhibited similar locomotion profiles during the recordings [Two-way repeated measures ANOVA, shNT, upper panel: time $\mathrm{x}$ brain region effect N.S., time main effect: N.S., brain region main effect: N.S., ACC n=5, CA1 n=5; shRNA lower panel: time $x$ brain region effect: N.S., time main effect: N.S., brain region main effect $p=$ N.S., ACC n=5 mice, CA1 $n=6$ mice] (Figure 1i). These data suggest that increasing FFI in DG-CA3 changes neuronal activity patterns in downstream CA1 and ACC in learning-naïve mice.

\section{Increasing FFI in DG-CA3 promotes foot-shock associated neuronal responses in CA1 and ACC during memory encoding.}

On day 0, control and experimental groups of mice were trained to associate context A with foot-shocks. Behavioral analysis of freezing levels revealed that both groups of mice responded similarly to foot-shocks in the training context A (Figure 2a, c). Consistent with prior work (Jimenez et al., 2020; Pan et al., 2020), our recordings of calcium transients during contextual fear conditioning revealed a subset of neurons in both CA1 and ACC that exhibited significant elevation in activity in response to foot-shocks (Foot-shock responsive neurons or FSR neurons) in both groups of mice [Two-tailed paired t-test, ACC shNT preFS vs postFS: $\mathrm{p}=0.004$, $\mathrm{n}=5$, ACC shRNA preFS vs postFS: $\mathrm{p}=0.013, \mathrm{n}=5$, CA1 shNT preFS vs postFS: $\mathrm{p}=0.007, \mathrm{n}=5$, CA1 shRNA preFS vs postFS: $\mathrm{p}=0.024, \mathrm{n}=5]$ (Figure $\mathbf{2 b}, \mathbf{d}, \mathbf{e})$. However, the fraction of FSR neurons in ACC was significantly increased in the shRNA group compared to shNT group [Two-tailed unpaired t test with Welch's correction, $\mathrm{p}=0.043, \mathrm{n}=5$ ] (Figure 2f, Supplementary Figure 1a). Further analysis of FSR neurons revealed that the increase in FSR neuron activity in CA1 and ACC was accompanied by an increase in co-activity of these neurons i.e increase in number of FSR correlated pairs [Two-tailed paired t-test, ACC shNT: $\mathrm{p}=0.021, \mathrm{n}=5$, ACC shRNA: N.S., n=5, CA1 shNT: p=0.021, n=5, CA1 shRNA: p=0.001, n = 5] (Figure 2g-h and Supplementary Figure 1b). Note, this was not seen in ACC for shRNA group presumably because of high activity levels prior to footshocks. Shuffled data indicate that the increase in correlated pairs was mostly due to increased number of calcium events. Additionally, the shRNA mice exhibited a significantly higher increase in correlated neurons in CA1 mice [Two-tailed unpaired t test with Welch's correction, $\mathrm{p}=0.044, \mathrm{n}=5$ ] (Supplementary Figure 1b). We did not detect increased co-activity of FSR with nonFSR neurons in CA1 or ACC [One-sample t-test against 0; ACC shNT: N.S, n=5, ACC shRNA: N.S., n=5, CA1 shNT: N.S., n=5 mice, CA1 shRNA: N.S. n=5].

Together these data suggest that increased DG-CA3 FFI facilitates foot-shock-induced neuronal responses and FSR ensemble-like activity in CA1 and ACC through increased recruitment of FSRs and synchronous activity of FSRs (Figure 2i).

\section{FFI in DG-CA3 facilitates emergence of training context-specific ensembles in CA1 and ACC following} learning

We next asked how increasing FFI modifies CA1 and ACC ensemble properties during recent recall of contextual fear memory. One day following fear conditioning, mice were returned to the conditioned context A and a novel, 
1 neutral distinct context C (Figure 1a,d and Figure 3a). Behavioral analysis revealed that both shRNA and shNT 2 groups exhibited high levels of discrimination of the two contexts and showed similar levels of freezing in training 3 context $\mathrm{A}$ and negligible levels of freezing in context $\mathrm{C}$ [Two-way repeated measures ANOVA, treatment $\mathrm{x}$ time 4 effect N.S., time main effect $\mathrm{p}=0.014$; treatment main effect N.S.; $n=10$, pooled ACC and CA1 mice] (Figure 5 3b). In context $A$, we observed synchronous activity, a learning-induced network property (Gonzalez et al., 2019; Liu et al., 2017; Modi et al., 2014; Rajasethupathy et al., 2015), suggestive of a context-associated ensemble in CA1. Specifically, both virus groups showed significantly increased numbers of correlated neurons compared to baseline levels only in context $\mathrm{A}$ and not in the novel, distinct neutral context $\mathrm{C}$ [one sample t test against 1 (BL), context A, shNT: $p=0.003, n=5$, shRNA: $p=0.055, n=5$; context C: shNT: N.S., $n=5$, shRNA: N.S., n=5] (Figure3c-d). Additionally, in context A, the shRNA group also showed a significant increase in number of correlated pairs in ACC indicative of emergence of a training context-associated ensemble in ACC [one sample $t$ test against 1 (BL), context A, ACC, shNT: N.S., shRNA: p=0.018, n=5] (Figure 3c-d). Using CellReg (Sheintuch et al., 2017) to register neurons across sessions, we found no reactivation of FSR neurons among the correlated pairs of neurons identified in CA1 in the recall session [shNT: mean: 0.0 S.E.M. 0; shRNA: mean 0.0, S.E.M, 0] (data not shown). We controlled for the possible effect of locomotion on neuronal activity by quantifying the time mice moved in each context and found no correlation between the number of correlated neuronal pairs and the time mice moved in the respective context [Pearson's R ${ }^{2}$, ACC context A, 0.19, ACC context C: 0.00, CA1 context A: 0.09, CA1 context C: 0.04, n=5 mice per group] (Supplementary Figure 2ad).

Analysis of constituent cell properties within recorded cell populations revealed that the majority of neurons were active in only one context with a small subset (CA1, shNT 15.4 \pm 1.9 , shRNA 14.1 \pm 2.2 ; ACC, shNT $23.7 \pm 2.4$, shRNA $14.5 \pm 4.6$; percentage of A\&C neurons expressed as mean \pm S.E.M) of neurons active in both contexts (hereafter referred to as "A\&C neurons") (Figure 3e-h). We used a normalized jaccard similarity index (Ahmed et al., 2020), which corrects for differences in number of overall recorded neurons per session to calculate the average number of A\&C neurons per day. Neurons in CA1 (including A\&C neurons) showed higher activity i.e. rate of calcium transients in context $\mathrm{C}$ relative to context $\mathrm{A}$ [Two-way ANOVA with repeated measures, CA1, shNT: context $x$ time effect $p<0.0001$, time main effect $p=0.007$, context main effect $p=0.028$, $\mathrm{n}=5$; shRNA, : context $\mathrm{x}$ time effect $\mathrm{p}<0.0001$, time main effect $\mathrm{p}=0.012$, context main effect $\mathrm{p}=0.029, \mathrm{n}=5$ ] [A\&C neurons: one sample $t$ test against $0, C A 1, \operatorname{shNT}: p=0.007$, shRNA: $p=0.048, n=5]$ suggestive of a novelty response (Supplementary Figure 2i-j, Figure3f). Interestingly, the shRNA group showed significantly fewer $\mathrm{A} \& \mathrm{C}$ neurons in CA1 indicating that increasing FFI in DG-CA3 reduced the number of neurons that were active in both contexts [Two-tailed unpaired t test with Welch's correction, $p=0.048, n=5$ ] (Figure 3h).

Thus, early stages of memory consolidation are characterized by increased co-activity of neurons within CA1 as also observed previously (Ognjanovski et al., 2017) and FSR neurons found during encoding do not appear to participate in CA1 context-specific ensembles at recent recall. Furthermore, increasing FFI in DG-CA3 facilitated the emergence of training context-specific ensembles in CA1 and ACC consistent with increased SWRspindle coupling between CA1-ACC during memory consolidation (Maingret et al., 2016; Ognjanovski et al., 2017; Steadman et al., 2020; Xia et al., 2017).

\section{FFI in DG-CA3 promotes stability and context specificity of CA1-ACC ensembles at remote timepoint}

Neuronal ensembles are thought to re-organize over time in the ACC as memories transition from a recent to a remote state. To determine how FFI in DG-CA3 influences properties of neuronal ensembles over time, we returned mice to the conditioned context $\mathrm{A}$ or neutral context $\mathrm{C}$ at 16 days following training and recorded neural 
activity in CA1 and ACC and measured freezing behavior (Figure 3i,j). Behavioral analysis revealed that the shRNA group exhibited significantly higher levels of discrimination between the two contexts than the shNT group [Two-way repeated measures ANOVA, treatment $x$ time effect N.S., treatment main effect: $p=0.028, n=10$ ] (Figure 3j). Both groups of mice maintained an increase in correlated pairs in CA1 during recall in context A, however the shRNA group did not exhibit a significant decrease in correlated pairs from day 1 to day 16 as seen for shNT group (Two-tailed paired t-test, shNT $\mathrm{p}=0.041$, shRNA: N.S. $\mathrm{n}=5$, comparison not shown]). Furthermore, the shRNA group showed increased co-activity in context $\mathrm{C}$ [One-sample t test against 1 (baseline, BL), context A, shNT, $p=0.006$; shRNA, $p=0.04$; context $\mathrm{C}$, shNT $\mathrm{p}=\mathrm{N}$.S .; shRNA, $\mathrm{p}=0.026$; $\mathrm{n}=5$;](Figure 3k).

Next, we analyzed A\&C neurons in CA1 and ACC. We found that the A\&C neurons in CA1 in the shRNA group, but not shNT group, exhibited higher activity (rate of calcium events) in context $\mathrm{C}$ relative to context $\mathrm{A}$ [one sample $t$ test against 0, shNT: N.S., shRNA: $p=0.012, n=5$ ] (Figure 31). This elevation in activity was peculiar to $A \& C$ neurons in shRNA group and was not detected in the overall activity of CA1 neurons in each context as seen on day 1 [Two-way ANOVA with repeated measures, CA1, shNT, context x time effect p ,0.0001, context main effect N.S., time main effect: $p=0.0004$, shRNA, context $x$ time effect $p<0.0001$, context main effect N.S., time main effect $\mathrm{p}=0.005, \mathrm{n}=5$ ] (Supplementary Figure 2k-l). Furthermore, the shRNA group showed significantly fewer A\&C neurons among all correlated pairs of neurons in CA1 compared to the shNT group [two tailed unpaired t test with Welch's correction, $p=0.017, n=5$ ] (Figure 3n and Supplementary Figure $\mathbf{2 g - h}$ ). While there was no difference in the number of A\&C neurons between virus groups in CA1 [two tailed unpaired t test with Welch's correction, N.S., $n=5$ ], the shRNA group showed significantly fewer A\&C neurons in ACC indicating that fewer neurons were active in both contexts $C$ [two-tailed unpaired t test with Welch's correction, $\mathrm{p}=0.028, \mathrm{n}=5$ ] (Figure 3m). The control group showed an increase in the number of A\&C neurons over time (Two-way repeated measures ANOVA, treatment $x$ time effect $\mathrm{p}=0.017$, Sidak's multiple comparison test day 1 vs day 16 , shNT p=0.039, shRNA p=0.405, comparison not shown).

Given that identification of A\&C neurons may vary based on the approach used to register cells (Sheintuch et al., 2017), we tested if the certainty of registration or the method of registration accounted for the virus-mediated effects that we found. We found no significant correlation between the registration certainty of the mice included in this study and the number of A\&C neurons that could explain our virus-mediated effects (Pearson's R ${ }^{2}$, shNT, ACC, 0.09, CA1, 0.05, shRNA, ACC, 0.129, CA1, 0.019) (Supplementary Figure 3a-b). When we used a fixed threshold, instead of a probability model, to register cells across sessions, we were able to reproduce the virusmediated differences seen in CA1 at a recent time point as well as in ACC at the remote timepoint demonstrating the robustness of our findings (Supplementary Figure 3c).

Together, these data suggest that increasing FFI in DG-CA3 prevents time-dependent decay of training context- associated ensemble in CA1, promotes formation of a neutral context-associated ensemble in CA1 and emergence of context-specific ensembles in ACC.

\section{A\&C neuron activity in CA1 is sufficient to accurately predict context}

Our analysis of CA1 and ACC ensembles at recent and remote timepoints suggests a sequential establishment of context specific ensembles in CA1 followed by formation of context specific ensembles in ACC at remote timepoint. While the majority of neurons were found to be only active in one context, our data suggest that the activity of $\mathrm{A} \& \mathrm{C}$ neurons in CA1 may also convey context-specific information. (Figure $\mathbf{3} \mathbf{f}, \mathbf{l}$ ). Therefore, we asked whether the activity of A\&C neurons in CA1 is sufficient for a decoder to predict context. A support vector 
machine trained on time-binned events $(5 \mathrm{sec})$ in A\&C neurons in CA1 performed better than shuffled data (see example distribution in Figure 4a). At the recent time point, day 1, calcium events in A\&C neurons in both groups were sufficient to reliably predict context [One-sample t test against 50 (chance level), shNT, $p=0.012$, shRNA, $\mathrm{p}=0.005, \mathrm{n}=4]$. The shRNA group maintained a similarly high level of information in the A\&C neurons as we were able to predict the context with similar accuracy at the remote time point [One-sample $t$ test against 50 (chance level), shRNA, $\mathrm{p}=0.008, \mathrm{n}=4]$. In contrast, decoder trained on shNT mice showed a reduction in prediction accuracy for the remote time point resulting in significantly less prediction accuracy in shNT mice compared to shRNA mice [Mann Whitney test, $\mathrm{p}=0.029, \mathrm{n}=4$ ] (Figure 4b). Nonetheless, the prediction accuracy in shNT mice maintained a significant-above-chance performance despite the fact that A\&C neurons did not show an elevation in activity at the remote time point (Figure 31) [One-sample $t$ test against 50, $s$ NT, $p=0.003, n=4$ ] (Figure 4b) supporting that both activity levels and synchronous firing are likely determinants of context specificity.

\section{Formation, stabilization and specificity of context-associated ensembles}

Our longitudinal imaging studies reveal evolving dynamics of ensemble properties in CA1-ACC networks underlying recent and remote memories and demonstrate how enhancing FFI in DG-CA3 impacts these properties to promote memory consolidation. Prior to learning we found that increasing FFI in DG-CA3 changed CA1ACC activity as captured in cross-region comparisons. Learning induced the formation of neuronal ensembles of foot-shock-responsive neurons in both ACC and CA1 and FFI potentiated this property in CA1. During early stages of memory consolidation (recall of recent memory), we observed the emergence of training contextassociated ensembles (increased numbers of co-active neurons) in both CA1 and ACC. Increasing FFI in DGCA3 promoted emergence of training context-associated ensemble in ACC suggestive of increased CA1-ACC communication (Figure 4c). Additionally, we observed a FFI dependent reduction in the number of neurons active in both training and neutral contexts in CA1(Figure 4d, top) reflecting increased specificity of neuronal ensembles. At remote recall, we observed that FFI in DG-CA3 prevented time-dependent decay of the training context-associated ensemble and promoted acquisition (or maintenance since it may have emerged prior to test at remote timepoint) of a neutral context specific ensemble in CA1 (Figure 4c, d). Within the ACC, and not evident at the recent timepoint, we observed a FFI dependent reduction in the number of neurons active in both training and neutral contexts (Figure 4d, bottom).

\section{Discussion}

The DG-CA3 circuit contributes to encoding of episodic memories conjunctive representations that relate people, objects, events with the spatial and temporal contexts in which they occur. This is thought to be accomplished in DG-CA3 by integration of processed sensory information about our external world channeled from higher order cortices (perirhinal, postrhinal) via the entorhinal cortex and by decreasing interference between similar representations (Hainmueller \& Bartos, 2020). Distinct or updated detail-rich contextual representations in CA3 are transferred to CA1 enroute to prefrontal cortical sites (ACC, infralimbic and prelimbic) for memory consolidation. Although PV INs in CA1 and ACC are thought to mediate learning-induced synchronization of neuronal activity to stabilize memory ensembles and channel information across different regions (Buzsaki, 2015; Caliskan et al., 2016; Dupret et al., 2013; Fernandez-Ruiz et al., 2021; Gan et al., 2016; Ognjanovski et al., 2017; Stark et al., 2014; Xia et al., 2017), we know much less about how PV INs in DG-CA3 contribute to these ensemble dynamics during memory consolidation. 
To address this gap in our knowledge, we deployed a previously characterized molecular tool to increase dentate granule cell recruitment of PV IN mediated perisomatic inhibition onto CA3 in a way that mimics learning induced modifications of dentate granule cell-PV IN-CA3 connectivity (Guo et al., 2018). By combining this molecular approach to enhance FFI in DG-CA3 with longitudinal calcium imaging of neuronal ensembles, we identified PV INs in DG-CA3 as critical arbiters of ensemble stabilization and specificity in CA1-ACC networks during memory consolidation. Our approach affords a unique opportunity to understand how a defined inhibitory connectivity motif (dentate granule cell-PV IN-CA3), rather than PV INs in CA3 per se, contributes to evolving ensemble properties underlying memory consolidation. While optogenetic or chemogenetic manipulations of PV INs (in CA1) have illuminated their roles in regulation of synchronous activity and in SWR-spindle coupling, these manipulations target PV INs rather than a circuit motif in a wiring diagram and as such, do not address input-specific recruitment of PV INs in these processes. Here, we interpret our data on stability and specificity of ensembles in CA1 and ACC during memory consolidation through the lens of this inhibitory circuit motif in DGCA3.

Prior to learning, CA1 and ACC cross-region comparisons revealed a FFI-dependent increase in neural activity of CA1 neurons relative to ACC neurons. These data suggest that increasing dentate granule recruitment of perisomatic inhibition onto CA3 neurons may change synaptic weights and excitability in CA1 and ACC neurons to prime these circuits for learning. This is further supported by our finding of facilitated neuronal responses following hippocampal learning.

We found synchronous activity of principal neurons (captured in correlated pairs) indicative of neuronal ensembles in CA1 and ACC during training, recent and remote recall (Gonzalez et al., 2019; Liu et al., 2017; Modi et al., 2014; Rajasethupathy et al., 2015). In contrast to a prior report on FSR neurons in ventral CA1 that project to the basolateral amygdala (Jimenez et al., 2020), we did not observe recruitment of FSR neurons into training context-associated ensembles in CA1 during recent recall. This differential contribution of dorsal and ventral hippocampal FSR neurons to context-associated ensembles may reflect output connectivity (basolateral amygdala vs. other downstream circuits) and distinct roles of dorsal and ventral hippocampus in encoding spatial and non-spatial information (Fanselow \& Dong, 2010; Strange et al., 2014).

Our data demonstrate that increased FFI in DG-CA3 promotes neuronal ensembles in both CA1 and ACC. 2 Following contextual fear learning, we observed emergence of training context-associated ensembles in CA1 in both groups of mice but only mice with increased FFI in DG-CA3 exhibited a learning-induced ensemble associated with the training context in the ACC. Analysis of neuronal ensembles during remote recall demonstrated that increasing FFI in DG-CA3 prevented the decay of training context associated ensemble and promoted the emergence of a neutral context associated ensemble in CA1. Based on these observations and our prior work showing that increasing FFI in DG-CA3 decreases overlap between two context-associated ensembles in CA3 (Guo et al., 2018), we propose a testable model for how our manipulation increases synchronous activity of principal neurons in CA1 and ACC. We predict that increasing granule cell recruitment of perisomatic inhibition onto CA3 neurons imposes sparseness and facilitates coordinated spiking or burst firing of CA3 neurons thus reducing the likelihood of recruitment of the same CA3 neurons to distinct ensembles (Csicsvari et al., 2000; de la Prida et al., 2006; Gómez-Ocádiz et al., 2021; Mori et al., 2007; Neubrandt et al., 2017; Sasaki et al., 2018; Torborg et al., 2010). In turn, assemblies of CA3 neurons encoding different contexts entrain activity of downstream ensembles of CA1 principal neurons. Distinct ensembles in CA1 recruit local PV INs to synchronize their activity and SWRs (Choi et al., 2018; Csicsvari \& Dupret, 2014; Gan et al., 2016; Malerba et al., 2017; Ognjanovski et al., 2017; Stark et al., 2014). Consequently, these changes in network properties of CA1 facilitate 
transfer of information to ACC via SWR-spindle coupling for memory consolidation (Maingret et al., 2016; Xia et al., 2017).

Increasing FFI in DG-CA3 also enhanced specificity of context-associated ensembles in CA1 and ACC. Previous studies demonstrated that subsets of neurons are shared across ensembles when mice experience two distinct environments proximal in time (Cai et al., 2016; Rubin et al., 2015). We found a reduction in the number of neurons active in both contexts (A\&C neurons) in ACC and a smaller proportion of A\&C neurons within context-associated neuronal ensembles in CA1 at remote recall. These A\&C neurons may function as "schema" neurons in that they represent shared features (statistical regularities) across ensembles and facilitate the formation of new context-associated ensembles (Abdou et al., 2018; McKenzie et al., 2014; McKenzie et al., 2013; Tse et al., 2007). Increased neuronal excitability has been shown to bias recruitment of neurons into ensembles (Yiu et al., 2014; Zhou et al., 2009). Higher activity of A\&C neurons (observed upon exposure to context C) may bias their allocation into the ensemble associated with the new context while maintaining context-specific activity through synaptic connectivity (Abdou et al., 2018; Gava et al., 2021). Indeed, we can train a decoder on activity of $\mathrm{A} \& \mathrm{C}$ neurons to predict context. Computational modeling has suggested that CA1 balances abstraction of statistical regularities across memories mediated by the temporoammonic pathway (EC->CA1) with encoding of detail-rich distinct contextual representations conveyed through the trisynaptic EC->DG->CA3->CA1 circuit (Schapiro et al., 2017). Our data supports this model since we observe a reduction in neurons encoding overlapping features (A\&C neurons) in CA1 (this study) when we increase FFI in DG-CA3 and reduce interference between context associated ensembles in CA3 (Allegra et al., 2020; Guo et al., 2018; Koolschijn et al., 2019a; Leutgeb et al., 2004). Thus, our manipulation biases the trade-off in CA1 towards generation of context-specific ensembles in CA1 over generation of schema.

Our data builds on foundational theories (McClelland et al., 1995; Nadel \& Moscovitch, 1997; Teyler \& DiScenna, 1986; Winocur et al., 2010; Winocur et al., 2007) to suggest a continuous role for CA1 in playing an instructive teacher-like role in governing re-organization of ensembles in ACC during memory consolidation. During memory consolidation, conjunctive representations are thought to be transformed into more schema like or gist-like structures necessary for cognitive flexibility and new learning. However, the extent to which memories retain details may be determined by the hippocampus. Increasing FFI in DG-CA3 appears to facilitate re-organization of ensembles in ACC and decrease the number of A\&C neurons (potentially, schema neurons) at the remote timepoint. Although in this study's experimental design, our behavioral analysis did not capture timedependent generalization of the contextual fear memory, mice with increased FFI in DG-CA3 exhibited significantly greater long-term memory than controls. We infer from our data that stabilization of trainingcontext associated ensembles in CA1 over time governs a toggle switch in the ACC that determines the extent to which memories may retain details over time. Such an interpretation is consistent with hippocampal indexing theory in that the maintenance of the hippocampal index for an experience permits access to details of the original experience stored in distributed neocortical sites (Besnard \& Sahay, 2016; Goode et al., 2020; Koolschijn et al., 2019b; Teyler \& DiScenna, 1986).

A major challenge in neuroscience is to assign functional significance to circuit motifs in wiring diagrams. Here, we build on our past work to demonstrate how the identification of learning-induced molecular regulators of connectivity may illuminate the relationship between a DGC-PV IN-CA3 circuit motif with emergent network properties during memory consolidation. The discovery of other molecular specifiers of principal neuroninhibitory neuron connectivity in combination with a "bottom-up synapse to systems approach" as conveyed here is likely to generate new fundamental insights into the physiological relevance of distinct hippocampal inhibitory 
0 circuit motifs in memory processing. It is plausible that connectivity re-engineering strategies such as that 1 described in this study harbor potential to enhance memory consolidation in aging and in mouse models of 2 Alzheimer's disease (McAvoy \& Sahay, 2017).

5 We thank members of Sahay lab, Liron Sheintuch and Yaniv Ziv for comments on the manuscript. H.T. was 6 supported by a German Research Foundation (DFG) Postdoctoral fellowship and is recipient of a Harvard Brain 7 Initiative Travel Grant. V.S was recipient of a Harvard Medical School Bertarelli Program in Translational 8 Neuroscience and Neuroengineering Masters research fellowship. A.S. acknowledges support from US National 9 Institutes of Health Biobehavioral Research Awards for Innovative New Scientists (BRAINS) 1-R01MH104175, 0 NIH-NIA 1R01AG048908-01A1, NIH 1R01MH111729-01, the James and Audrey Foster MGH Research 1 Scholar Award, Ellison Medical Foundation New Scholar in Aging, the Inscopix Decode Award, Ellison Family 2 Philanthropic support, Blue Guitar Fund, Alzheimer's Association research grant and The Simons Collaboration 3 on Plasticity and the Aging Brain. 


\section{Material \& Methods}

Animal care. All mice were group housed in a $12 \mathrm{~h}(7 \mathrm{am}$ to $7 \mathrm{pm}$ ) light/dark cycle at room temperature with ad libitum access to food and water. After surgery, mice were split into pairs and housed with a divider to protect the implant without single housing mice. Male, 2-3 months old C57B16 mice were obtained from Jackson Laboratories. All animals were handled, and experiments were conducted in accordance with procedures approved by the Institutional Animal Care and Use Committee at the Massachusetts General Hospital and Boston University in accordance with NIH guidelines.

Lenti virus production: lenti-shNT and lenti-shRNA virus were generated in the lab using previously described pLLX-shRNA lentiviral vectors (Guo et al,2018) or a similar lentivirus U6-based shRNA knockdown vector from VectorBuilder. Expression of GFP or mCherry under the ubiquitin C promoter allowed to monitor the efficiency of transfection and infection both during virus production as well as in in vivo experiments. HEK293T cells were transfected with lentiviral vector and packaging plasmids, VSVG and $\Delta 8.9$, as described previously (Guo et al., 2018; Lois et al., 2002) to generate lenti-virus stock solutions. We used Lenti-X GoStix Plus (Takara Bio USA) to quantify the titer of each virus batch used in this study. The generated virus was further validated both in vitro by infection HEK293T cells and in vivo by injection into the hippocampus of 2-3 month old male C57BL6 mice. All virus stock solutions used in this study ranged from $200-300 \mathrm{ng} / \mathrm{ml} \mathrm{p} 24$. Virus aliquots were stored at $-80^{\circ} \mathrm{C}$ for a maximum of 12 month and not reused (no freeze-thaw cycle).

Virus injections \& stereotactic surgeries: Prior to surgery mice received an injection of carprofen $(5 \mathrm{mg} / \mathrm{kg}$, subcutaneously, Patterson Veterinary Supply). Mice were anaesthetized with ketamine and xylazine $(10 \mathrm{mg} / \mathrm{mL}$ and $1.6 \mathrm{mg} / \mathrm{mL}$, intraperitoneally, Patterson Veterinary Supply) and placed at a planar angle in a stereotaxic frame (Stoelting). Eyes were protected with Puralube (Dechra). After exposing the skull, craniotomies above the target site for injections were created using a Foredom K.1070 High Speed Rotary Micromotor Kit. The injection was performed with a hamilton microsyringe (Hamilton, Neuros Syringe 7001) that was slowly lowered into the target location. For virus validation and calcium imaging of ACC neurons, bilateral lenti-virus injections into the DG were performed using following coordinates: $-1.9 \mathrm{~mm}(\mathrm{AP}),+/-1.35 \mathrm{~mm}(\mathrm{ML}),-2.2 \mathrm{~mm}(\mathrm{DV})$ relative to bregma. For calcium imaging of CA1 neurons, we used an angle to access DG without damaging overlaying CA1. We adjusted the manipulator arm to an angle of $-20^{\circ}$ and used the following coordinates: $-1.1 \mathrm{~mm}(\mathrm{AP}),+/-1.4 \mathrm{~mm}$ (ML), $-2.3 \mathrm{~mm}$ (DV) relative to bregma. A total of $0.5 \mu 1$ virus solution (non diluted) was injected per hemisphere.

The calcium indicator GCaMP6f was unilaterally injected using an AAV1.CaMKII.GCaMP6f.WPRES.SV40 virus (Penn Vector Core) in either ACC (+1.0mm (AP), $-0.35 \mathrm{~mm}$ (ML), -2.0/-1.6 (DV)) or CA1 (-1.9mm (AP), $-1.4 \mathrm{~mm}$ (ML), $-1.6 \mathrm{~mm}$ (DV). For ACC, we spread the injection across two depth to achieve a better distribution of the virus. A total of $0.5 \mu 1$ at a 1:5 dilution was injected. Mice that underwent calcium imaging in CA1, received first the GCaMP6f injection in CA1 and three days later the angled lenti-virus injection into the DG. Mice with ACC imaging received both injections on the same day. At least 10 minutes after injection, needles were removed, and the skin incision closed with coated vicryl sutures (Ethicon US LLC). Mice received a daily injection of carprofen ( $5 \mathrm{mg} / \mathrm{kg}$, subcutaneously, Patterson Veterinary Supply) for three days following surgery.

Lens implantation: At least one week after the last virus injection, mice were implanted with a GRIN lens $(0.5 \mathrm{~mm} \varnothing \times 4 \mathrm{~mm}$ in ACC, $1 \mathrm{~mm} \varnothing \times 4 \mathrm{~mm}$ in CA1). We applied the same analgesia and anesthesia strategy as 
described above. Mice were placed in the stereotaxic frame and fixed at a planar angle. The skull was exposed, and a craniotomy created above the target site (ACC: $+0.9 \mathrm{~mm}$ (AP), $-0.3 \mathrm{~mm}(\mathrm{ML}),-1.3 \mathrm{~mm}(\mathrm{DV})$; CA1 -1.85mm (AP), $-1.35 \mathrm{~mm}$ (ML), -1.4mm (DV)). In addition, we created two small craniotomies to insert anchor screws (Basi Bioanalytical Systems). For CA1, we removed the overlaying cortex, as described Kinsky et al (Kinsky et al., 2020) using blunt needles of decreasing diameter (25ga to 31ga, SAI Infusion Technologies) attached to a vacuum pump while constantly applying cooled, sterile saline. A microscope (Nikon SMZ800) was placed above the stereotaxic frame to allow visual control of the procedure. Once the cortex was removed and possible bleeding stopped with gelfoam (Pfizer), the lens was attached to a custom-made lens holder and slowly lowered into the craniotomy. Coordinates for the lens were referenced to bregma. For ACC, no aspiration was performed. Instead, we inserted a $30 \mathrm{ga}$ needle at the ACC coordinates (AP \& ML) and slowly lowered it $-1.0 \mathrm{~mm}$ DV into the cortex to open the track for the lens. After 10min the needle was slowly removed and the GRIN lens, attached to a custom-made holder, inserted into ACC. Once the lens in either brain region was inserted, the gap between lens and skull was sealed with superglue before the lens was cemented to the skull with dental cement (Stoelting). A thin layer of blackened dental cement (by adding nail polish to the cement) was applied as final layer to shield the lens from light. The remaining part of the lens was covered with Kwik-cast sealant (World Precision Instruments).

One week following the lens implant, mice were placed back in the stereotaxic frame as described above. The Kwik-cast sealant was removed from the lens and possible residues cleaned off with lens paper. A baseplate was attached to the microscope (Inscopix) and positioned above the lens. The field of view (FoV) was visualized with nVista HD software (Inscopix) and the microscope carefully adjusted to be parallel to the lens surface. The microscope was lifted towards the best possible visualization of vasculature and putative neurons (approx. 200 to $250 \mu \mathrm{m}$ above the focus of the lens surface). Before applying the dental cement, the microscope was raised another $50 \mu \mathrm{m}$. The baseplate was firmly attached to the previous implant with dental cement and a final layer of blackened cement was again applied to shield the lens from light. Once the cement hardened, we removed the microscope and attached a baseplate cover (Inscopix).

Behavioral testing: Mice were tested in a series of behavioral paradigms while calcium imaging was performed. Videos were acquired with a CCD camera (OMRON SENTECH) at 30 frames/second. Prior to any experiment, mice were allowed to rest in a quiet holding area for at least 1h. Each day, mice would first undergo a 6 minute recording in a familiar context (Makrolon cage with bedding, baseline recording). After 2 hours mice were tested in an open field arena (OF) (Plexiglas box, $41 \times 41 \mathrm{~cm}$ (Kinder Scientific) for $15 \mathrm{~min}$ (day -3 ), an elevated plus maze (gray taped Plexiglass, $16 \mathrm{~cm}$ x $5 \mathrm{~cm}$ arms with 2 open arms and 2 arms with vertical walls $1 \mathrm{~m}$ above ground) for 6 minutes (day -2) and a contextual fear conditioning paradigm (day -1 to day 16). For the contextual fear paradigms, we used 2 different contexts. Context A consisted of a squared conditioning chamber $(18 \times 18 \times 30$ $\mathrm{cm}$ ) with two metal and two clear walls and a stainless-steel grid floor (Coulbourn Instruments). Context $\mathrm{C}$ consisted of a round, white coated paper bucket with blue stripes $(18 \varnothing \times 30 \mathrm{~cm})$. Each recording was performed for 6 minutes. Mice were pre-exposed to context A without a foot-shock on day-1 (preA). One day later, mice were re-introduced to context $\mathrm{A}$ and received three foot-shocks $(2 \mathrm{~s}, 0.75 \mathrm{~mA})$ at $180 \mathrm{sec}, 240 \mathrm{sec}$ and $300 \mathrm{sec}$ (training day, day 0). The next day, mice were tested in context $\mathrm{A}$ and 2 hours later in context $\mathrm{C}$ (day 1). The days after training in context A (day 16). 
7 Quantification of behavior: Behavioral videos were exported as AVI from Freezeframe (Actimetrics) and 8 analyzed with Ethovision 15 (Noldus). Freezing behavior was quantified using the activity feature that is based 9 on changes in pixel and therefore more robust against artefacts from the cable. Moving was measured as motion 0 of the body center point.

2 Calcium imaging acquisition: Mice were allowed to recover at least a week before being handled. Once mice 3 were recovered, they were handled using the tunnel handling approach (see https://www.nc3rs.org.uk/mouse4 handling-poster) and habituated to the microscope. All recordings were performed using nVista HD (Inscopix). 5 Prior to recording sessions, mice were tested for sufficient calcium signal in the FoV, a reference image was 6 taken, and the LED intensity tested to be optimal for recording. The LED intensity for each animal was maintained 7 throughout the experiment. During the behavior paradigm, the microscope (nVista2, Inscopix) was attached to 8 the baseplate and, when necessary, the FoV adjusted to match the reference image. Average number of recorded 9 neurons per mouse and session were as follow: CA1: shNT mean:137, S.E.M. 15, shRNA mean:161, S.E.M. 20, ACC: shNT mean:89, S.E.M 21, shRNA mean: 68, S.E.M 21. Videos were recorded at 20 frames/second and a resolution of $1440 \times 1280$ pixels. We used a TTL-pulse to align the onset of behavioral and calcium video recording.

3 Perfusion \& histology: Mice were perfused with 4\% PFA after completion of the behavioral paradigm. Brains 4 were stored overnight in $4 \%$ PFA at $4^{\circ} \mathrm{C}$ before being placed in $30 \%$ sucrose solution for 3 days at $4{ }^{\circ} \mathrm{C}$. Brains 5 were embedded in Optimal Cutting Temperature medium (OCT, Fisher HealthCare) and stored at $-80^{\circ} \mathrm{C}$. 6 Histological coronal sections $(35 \mu \mathrm{m})$ were generated with a Leica cryostat (Leica) and stored in PBS with $0.01 \%$ 7 sodium azide at $4{ }^{\circ} \mathrm{C}$. Sections were immunohistologically stained as follow: Free floating sections were washed 8 in PBS triton (0.3\%) and unspecific binding sides blocked with blocking buffer (10\% Normal Donkey Serum in 9 PBS $0.3 \%$ Triton) for 2 hours at room temperature. Sections were further incubated with primary antibodies (as 0 specified below) diluted in PBS $0.3 \%$ triton overnight at $4{ }^{\circ} \mathrm{C}$. The next day, sections were washed 3 times $(10$ $1 \mathrm{~min}$ ) in PBS and incubated with secondary antibodies (as specified below) diluted in PBS for $2 \mathrm{~h}$ at room 2 temperature.

3 To validate the expression of the lenti-virus in all mice included in this study, we stained sections using antibodies 4 against the fluochrome expressed by the lenti-virus (GFP or mCherry).

5 To quantify the effect of lenti-virus injection on inhibition, we used PV puncta as a histological marker as 6 demonstrated previously (see Guo et al). See table for primary antibody details. We used alexa-fluor secondary 7 antibodies (donkey, Jackson Immuno Research, 1:500 dilution) targeted against the host of the primary 8 antibody.

\begin{tabular}{|l|l|l|l|}
\hline Target & Antibody (primary) & Fluor of 2nd AB & Purpose \\
\hline GFP & Chicken anti-GFP (Aves, GFP-1020, 1:2000) & 488 & $\begin{array}{l}\text { Label lenti-virus } \\
\text { expression }\end{array}$ \\
\hline mCherry & $\begin{array}{l}\text { Rabbit anti-RFP (Rockland, 600-401-379, } \\
1: 200)\end{array}$ & $\begin{array}{l}\text { Label } \\
\text { expression }\end{array}$ \\
\hline
\end{tabular}




\begin{tabular}{|l|l|l|l|}
\hline Parvalbumin & $\begin{array}{l}\text { Mouse anti-PV (EMD Millipore, MAB 1572, } \\
1: 2000)\end{array}$ & Quantify PV puncta \\
\hline
\end{tabular}

PV quantification: We quantified the level of PV puncta in dorsal CA3, dorsal CA1 and ACC. For that, we used a confocal microscope (Leica TCS SP8) with 60x oil objective to generate high-resolution images. Images were acquired using a sequence to reduce cross-talk. We imaged 18 ROIs per mouse (3 brain sections, 3 ROIs per hemisection). We used a zoom of $4 \mathrm{x}$ for CA3, and 2x for CA1 and ACC. Images were blinded with Image randomizer macro (macro by Tiago Ferreira) and scored manually. In CA1, we distinguished between superficial and deep layer by drawing a $20 \mu \mathrm{m}$ deep square from either the upper (deep layer) or lower (superficial) border of dense DAPI signal.

Calcium video processing: Videos were decompressed and preprocessed with Inscopix Data Processing Software (Inscopix) using the motion correction and temporal down sampling to $10 \mathrm{~Hz}$. Preprocessed videos were exported as TIFF and further analyzed with MATLAB 2018a (Mathworks).

Spatial and temporal calcium signal extraction: We used CNMF-E (Zhou et al., 2018) to extract spatial and temporal traces of individual neurons. We developed a MATLAB based code to further filter the extracted traces from CNMF-E to ensure, to the best of our ability in larger data sets, that our analysis is free from artefacts such as noise. We did so by removing: 1) possible false positive neurons based on contour features, 2) possible false positive (noisy) calcium events, 3 ) neurons with low activity after removing noisy events, 4) low signal neurons with high overlapping neighbor neurons.

We removed elongated shapes using the polygeom function. If the inertia ratio was larger than 5, we rejected the neuron to exclude possible dendrites or blood vessels or visually distorted neurons. Furthermore, we removed neurons with rough contours that likely stemmed from background fluorescence using a simple linear classifier. The roughness, called compacity in this case, is calculated as:

$$
C=\frac{P^{2}}{4 \pi A}
$$

where $\mathrm{C}$ is the compacity, $\mathrm{P}$ the perimeter and $\mathrm{A}$ the area of the neuron. The compacity threshold was empirically determined at 35. While CNMF-E includes a criteria to restrict overlap of neurons, we still found a small set of highly overlapping neurons in our extractions. To avoid a time-costly re-iteration of CNMF-E, we included this part of the CNMF-E approach into our pipeline. We identified candidate pairs with close proximity using an empirically tested threshold of 36 pixels between the center of each neuron. The overlap was then calculated as follow:

$$
\text { overlap }=\frac{1}{2}\left(\frac{\text { Area }_{N_{1} \cap N_{2}}}{\text { Area }_{N_{1}}}+\frac{\text { Area }_{N_{1} \cap N_{2}}}{\text { Area }_{N_{2}}}\right)
$$

where $\operatorname{Area}_{N_{1} \cap N_{2}}$ is the overlap area between the two neurons 1 and 2 and $A_{r e a_{N 1}} \&$ Area $a_{\mathrm{N} 2}$ the number of pixels of neurons 1 and 2, respectively. The area of neurons and the overlap area were calculated using the polyshape function. If neurons overlapped more than 50\%, the neuron with lower PNR was removed. 
To remove noisy calcium events, we used a 2-step approach. First, we extracted the timestamp of possible calcium events (event-candidates) using the findpeak function on the raw fluorescence traces and the denoised traces from CNMF-E (neuron.C_raw, neuron.C). A peak was defined by an amplitude of at least 3 SD from baseline noise, a minimum distance between two peaks of 15 frames (1.5 second) and a minimum duration of 3 frames (300ms). We further extracted 8 features of the event-candidates from both the raw and denoised fluorescence traces based on the findpeak function: Peak-to-noise ratio (PNR), Width-to-Height ratio, Peak prominence, Pearson correlation between peaks in the raw and denoised traces, and local PNR of the event candidate in the raw trace. For the local PNR, we calculated the standard deviation of the baseline in the vicinity of the peak rather than the overall recording baseline. We performed a PCA ( $p c a$ function) on those 8 parameters for dimension reduction to extract the components of these parameters that could be best used for classification. To reliable identify and remove calcium events that we considered noisy, we then trained a quadratic Bayesian classifier (fitcdiscr) on manually classified calcium peaks. After removing noise calcium events, neurons with low activity were removed using a linear classifier

$$
N_{\text {peaks }}+2 \times H_{\max }-3>0
$$

where $\mathrm{N}_{\text {peaks }}$ is the neuron's number of calcium events within one calcium recording and $\mathrm{H}_{\max }$ the relative height of the maximal calcium peak. We updated the CNMF-E output (neuron.A, neuron.C and neuron.C raw) to remove all neurons indicated by our pipeline and generated a file with calcium event times (sparse time series) for each neuron matching CNMF-E indexes. The updated data set was used for all further analysis presented in the figures.

Correlated pairs: We calculated the correlation of calcium events between neurons using the xcorr function on time series data. We added a 200msec frame around the calcium event (Barbera et al., 2016). We generated a distribution of correlation using temporally shuffled calcium event timeseries and calculated a threshold (mean+2SD) based on this distribution. Correlated pairs were defined as pairs with a correlation above this threshold. For analysis of foot-shock induced changes in cross-correlated pairs, we only included 30sec extracts of the preFS and postFS time series data (see Figure 2). To compare numbers of correlated pairs across sessions (e.g. between context $\mathrm{A}$ and context $\mathrm{C}$ ), we normalized the number of correlated pairs to the number of correlated pairs found in the same day baseline $\left(\mathrm{CC}_{\text {pairs ctx } \mathrm{A}} / \mathrm{CC}_{\text {pairs baseline }}\right)$.

FSR neurons: To identify FSR neurons, we z-scored the calcium activity (calcium events) of each neuron to its time-binned (5sec) activity (calcium events) prior to the foot-shock (Pan et al., 2020). Neurons that increased their activity above 2SD during or after any foot-shock were classified as FSR neurons. To further quantify the foot-shock response we used 30 sec timebin before and after the foot-shock (preFS vs postFS) and calculated the normalized activity before and after each foot-shock. We use the average of all 3 foot-shocks (preFS vs postFS) to quantify the overall effect of foot-shock training on those cells, which is why the average postFS response can be smaller than the initial threshold of 2SD [threshold to qualify as FSR].

Identification of A\&C neurons: We used CellReg (Sheintuch et al., 2017) to register neurons across different sessions (contexts and days). Spatial maps of neuronal contours were extracted by reshaping the spatial CNMFE output (neuron.A) and down sampled 4x for faster processing. We used two different approaches to calculate a threshold for same neurons and different neurons, one is the probabilistic approach based on the distribution of our data and the other is a fixed threshold (see (Sheintuch et al., 2017) for details). For all our recordings, spatial correlation was more reliable than centroid distance. We experienced that sessions with larger inter-session 
8 intervals (i.e. day 1 and day 16) were less accurate registered using the probabilistic approach than sessions that 9 occurred closely in time. Therefore, we used the probabilistic approach only on sessions that were maximally $24 \mathrm{~h}$ 0 apart (training day \& day 1; day 10; day16, data in main figures). To ensure that our findings were not driven by 1 the cell registration approach, we also registered neurons using a fixed spatial correlation threshold of 0.85 as that 2 was the average threshold we obtained in the probabilistic approach. This further allowed us to register neurons 3 across all sessions (training day to day 16) as shown in the supplementary figures. A\&C neurons were defined as 4 neurons that were active in both sessions (session A and session C) on the same day.

Quantification of A\&C neurons: The number of A\&C neurons was expressed as normalized jaccard similarity 7 index (Ahmed et al., 2020)

$$
J(A, C)=\mid \frac{|A \cap C|}{|A \cup C|}
$$

with $\mathrm{A}=$ neurons in session $\mathrm{A}, \mathrm{C}=$ neurons in session $\mathrm{C}$. The jaccard similarity index describes the number of $\mathrm{A} \& \mathrm{C}$ neurons relative to the total number of neurons active in $\mathrm{A}$ and $\mathrm{C}$. The number of $\mathrm{A} \& \mathrm{C}$ neurons however can be biased by the total number of neurons active during one session. Given that then pool of neurons that we record from is limited, recordings with larger number of neurons are stochastically more likely to contain A\&C neurons than recordings with smaller number of neurons. To correct for this bias, we generated a distribution of 1000 jaccard similarity indices by randomizing neuronal indexes while keeping the number of active neurons recorded per session. The jaccard index was then z-scored to that distribution. Furthermore, z-scored indices were normalized to their minimum and maximum to allow cross object comparison.

Decoding context: We trained a support vector using the MATLAB statistics and machine learning toolbox. To avoid overfitting, we only included mice that had at least $50 \mathrm{~A} \& \mathrm{C}$ neurons per day. Instead of withholding testing data, we used 10fold cross-validation to train and test our data. The decoder was based on time binned ( $5 \mathrm{sec}$ ) calcium event time series with averaged events per time bin of A\&C neurons for both sessions. Thus, we obtained 72 vectors per context with the length of all A\&C neuron reflecting each neuron's activity at a given time point. We used the decoder to test for differences between these vectors that could predict the context. For all mice, we generated 1000 shuffled data set of randomized calcium event time (maintaining the same number of total calcium events/neuron and neurons) to train the decoder and test prediction certainty. All randomized data sets resulted in chance level of prediction.

Statistics: Statistical tests were performed with GraphPad Prism v9 (Graphpad Software). We used nonparametric tests for sample sizes smaller than 5. For data sets with at least 5 mice, we used two-tailed students ttest (with Welch correction for unpaired data), one sample t-test (against 0, 1 or 50, see statistic table and figures for details) or two-way ANOVA with repeated measures and Sidak's multiple comparison test when appropriate. For all tests, significance was set at $\mathrm{p}<0.05$. Individual data points reflect the average per mouse. Number of mice per group are indicated by $n$. Details for all statistical analysis are provided in Supplementary table 1. 


\section{References}

Abdou, K., Shehata, M., Choko, K., Nishizono, H., Matsuo, M., Muramatsu, S. I., \& Inokuchi, K. (2018). Synapse-specific representation of the identity of overlapping memory engrams. Science, 360(6394), 1227-1231. https://doi.org/10.1126/science.aat3810

Ahmed, M. S., Priestley, J. B., Castro, A., Stefanini, F., Solis Canales, A. S., Balough, E. M., Lavoie, E., Mazzucato, L., Fusi, S., \& Losonczy, A. (2020). Hippocampal Network Reorganization Underlies the Formation of a Temporal Association Memory. Neuron, 107(2), 283-291 e286. https://doi.org/10.1016/i.neuron.2020.04.013

Allegra, M., Posani, L., Gomez-Ocadiz, R., \& Schmidt-Hieber, C. (2020). Differential Relation between Neuronal and Behavioral Discrimination during Hippocampal Memory Encoding. Neuron, 108(6), 1103-1112 e1106. https://doi.org/10.1016/i.neuron.2020.09.032

Barbera, G., Liang, B., Zhang, L., Gerfen, C. R., Culurciello, E., Chen, R., Li, Y., \& Lin, D. T. (2016). Spatially Compact Neural Clusters in the Dorsal Striatum Encode Locomotion Relevant Information. Neuron, 92(1), 202-213. https://doi.org/10.1016/i.neuron.2016.08.037

Besnard, A., \& Sahay, A. (2016). Adult Hippocampal Neurogenesis, Fear Generalization, and Stress. Neuropsychopharmacology, 41(1), 24-44. https://doi.org/10.1038/npp.2015.167

Buzsaki, G. (2015). Hippocampal sharp wave-ripple: A cognitive biomarker for episodic memory and planning. Hippocampus, 25(10), 1073-1188. https://doi.org/10.1002/hipo.22488

Cai, D. J., Aharoni, D., Shuman, T., Shobe, J., Biane, J., Song, W., Wei, B., Veshkini, M., La-Vu, M., Lou, J., Flores, S. E., Kim, I., Sano, Y., Zhou, M., Baumgaertel, K., Lavi, A., Kamata, M., Tuszynski, M., Mayford, M., Golshani, P., \& Silva, A. J. (2016). A shared neural ensemble links distinct contextual memories encoded close in time. Nature, 534(7605), 115-118. https://doi.org/10.1038/nature17955

Caliskan, G., Muller, I., Semtner, M., Winkelmann, A., Raza, A. S., Hollnagel, J. O., Rosler, A., Heinemann, U., Stork, O., \& Meier, J. C. (2016). Identification of Parvalbumin Interneurons as Cellular Substrate of Fear Memory Persistence. Cereb Cortex, 26(5), 2325-2340. https://doi.org/10.1093/cercor/bhw001

Choi, J. H., Sim, S. E., Kim, J. I., Choi, D. I., Oh, J., Ye, S., Lee, J., Kim, T., Ko, H. G., Lim, C. S., \& Kaang, B. K. (2018). Interregional synaptic maps among engram cells underlie memory formation. Science, 360(6387), 430-435. https://doi.org/10.1126/science.aas9204

Csicsvari, J., \& Dupret, D. (2014). Sharp wave/ripple network oscillations and learning-associated hippocampal maps. Philos Trans R Soc Lond B Biol Sci, 369(1635), 20120528. https://doi.org/10.1098/rstb.2012.0528

Csicsvari, J., Hirase, H., Mamiya, A., \& Buzsaki, G. (2000). Ensemble patterns of hippocampal CA3-CA1 neurons during sharp wave-associated population events. Neuron, 28(2), 585-594. https://doi.org/10.1016/s08966273(00)00135-5

de la Prida, L. M., Huberfeld, G., Cohen, I., \& Miles, R. (2006). Threshold behavior in the initiation of hippocampal population bursts. Neuron, 49(1), 131-142. https://doi.org/10.1016/j.neuron.2005.10.034

DeNardo, L. A., Liu, C. D., Allen, W. E., Adams, E. L., Friedmann, D., Fu, L., Guenthner, C. J., Tessier-Lavigne, M., \& Luo, L. (2019). Temporal evolution of cortical ensembles promoting remote memory retrieval. Nat Neurosci, 22(3), 460469. https://doi.org/10.1038/s41593-018-0318-7

Dupret, D., O'Neill, J., \& Csicsvari, J. (2013). Dynamic reconfiguration of hippocampal interneuron circuits during spatial learning. Neuron, 78(1), 166-180. https://doi.org/10.1016/j.neuron.2013.01.033

Fanselow, M. S., \& Dong, H. W. (2010). Are the dorsal and ventral hippocampus functionally distinct structures? Neuron, 65(1), 7-19. https://doi.org/10.1016/i.neuron.2009.11.031

Fernandez-Ruiz, A., Oliva, A., Soula, M., Rocha-Almeida, F., Nagy, G. A., Martin-Vazquez, G., \& Buzsaki, G. (2021). Gamma rhythm communication between entorhinal cortex and dentate gyrus neuronal assemblies. Science, 372(6537). https://doi.org/10.1126/science.abf3119

Frankland, P. W., \& Bontempi, B. (2005). The organization of recent and remote memories. Nat Rev Neurosci, 6(2), 119130. https://doi.org/10.1038/nrn1607

Frankland, P. W., Bontempi, B., Talton, L. E., Kaczmarek, L., \& Silva, A. J. (2004). The involvement of the anterior cingulate cortex in remote contextual fear memory. Science, 304(5672), 881-883. 
Gan, J., Weng, S. M., Pernia-Andrade, A. J., Csicsvari, J., \& Jonas, P. (2016). Phase-Locked Inhibition, but Not Excitation, Underlies Hippocampal Ripple Oscillations in Awake Mice In Vivo. Neuron. https://doi.org/10.1016/i.neuron.2016.12.018

Gava, G. P., McHugh, S. B., Lefèvre, L., Lopes-Dos-Santos, V., Trouche, S., El-Gaby, M., Schultz, S. R., \& Dupret, D. (2021). Integrating new memories into the hippocampal network activity space. Nat Neurosci, 24(3), 326-330. https://doi.org/10.1038/s41593-021-00804-w

Gonzalez, W. G., Zhang, H., Harutyunyan, A., \& Lois, C. (2019). Persistence of neuronal representations through time and damage in the hippocampus. Science, 365(6455), 821-825. https://doi.org/10.1126/science.aav9199

Goode, T. D., Tanaka, K. Z., Sahay, A., \& McHugh, T. J. (2020). An Integrated Index: Engrams, Place Cells, and Hippocampal Memory. Neuron. https://doi.org/10.1016/j.neuron.2020.07.011

Goshen, I., Brodsky, M., Prakash, R., Wallace, J., Gradinaru, V., Ramakrishnan, C., \& Deisseroth, K. (2011). Dynamics of retrieval strategies for remote memories. Cell, 147(3), 678-689. https://doi.org/10.1016/i.cell.2011.09.033

Guo, N., Soden, M. E., Herber, C., Kim, M. T., Besnard, A., Lin, P., Ma, X., Cepko, C. L., Zweifel, L. S., \& Sahay, A. (2018). Dentate granule cell recruitment of feedforward inhibition governs engram maintenance and remote memory generalization. Nat Med, 24(4), 438-449. https://doi.org/10.1038/nm.4491

Gómez-Ocádiz, R., Trippa, M., Posani, L., Cocco, S., Monasson, R., \& Schmidt-Hieber, C. (2021). A synaptic novelty signal to switch hippocampal attractor networks from generalization to discrimination. bioRxiv, 2021.2002.2024.432612. https://doi.org/10.1101/2021.02.24.432612

Hainmueller, T., \& Bartos, M. (2020). Dentate gyrus circuits for encoding, retrieval and discrimination of episodic memories. Nat Rev Neurosci, 21(3), 153-168. https://doi.org/10.1038/s41583-019-0260-z

Jimenez, J. C., Berry, J. E., Lim, S. C., Ong, S. K., Kheirbek, M. A., \& Hen, R. (2020). Contextual fear memory retrieval by correlated ensembles of ventral CA1 neurons. Nat Commun, 11(1), 3492. https://doi.org/10.1038/s41467-02017270-w

Kemere, C., Carr, M. F., Karlsson, M. P., \& Frank, L. M. (2013). Rapid and continuous modulation of hippocampal network state during exploration of new places. PLoS One, 8(9), e73114. https://doi.org/10.1371/journal.pone.0073114

Kinsky, N. R., Mau, W., Sullivan, D. W., Levy, S. J., Ruesch, E. A., \& Hasselmo, M. E. (2020). Trajectory-modulated hippocampal neurons persist throughout memory-guided navigation. Nat Commun, 11(1), 2443. https://doi.org/10.1038/s41467-020-16226-4

Kitamura, T., Ogawa, S. K., Roy, D. S., Okuyama, T., Morrissey, M. D., Smith, L. M., Redondo, R. L., \& Tonegawa, S. (2017). Engrams and circuits crucial for systems consolidation of a memory. Science, 356(6333), 73-78. https://doi.org/10.1126/science.aam6808

Koolschijn, R. S., Emir, U. E., Pantelides, A. C., Nili, H., Behrens, T. E. J., \& Barron, H. C. (2019a). The Hippocampus and Neocortical Inhibitory Engrams Protect against Memory Interference. Neuron, 101(3), 528-541 e526. https://doi.org/10.1016/i.neuron.2018.11.042

Koolschijn, R. S., Emir, U. E., Pantelides, A. C., Nili, H., Behrens, T. E. J., \& Barron, H. C. (2019b). The Hippocampus and Neocortical Inhibitory Engrams Protect against Memory Interference. Neuron, 101(3), 528-541.e526. https://doi.org/10.1016/i.neuron.2018.11.042

Leutgeb, S., Leutgeb, J. K., Treves, A., Moser, M. B., \& Moser, E. I. (2004). Distinct ensemble codes in hippocampal areas CA3 and CA1. Science, 305(5688), 1295-1298. https://doi.org/10.1126/science.1100265

Liu, Y. Z., Wang, Y., Shen, W., \& Wang, Z. (2017). Enhancement of synchronized activity between hippocampal CA1 neurons during initial storage of associative fear memory. J Physiol, 595(15), 5327-5340. https://doi.org/10.1113/JP274212

Lois, C., Hong, E. J., Pease, S., Brown, E. J., \& Baltimore, D. (2002). Germline transmission and tissue-specific expression of transgenes delivered by lentiviral vectors. Science, 295(5556), 868-872.

https://doi.org/10.1126/science.1067081

Maingret, N., Girardeau, G., Todorova, R., Goutierre, M., \& Zugaro, M. (2016). Hippocampo-cortical coupling mediates memory consolidation during sleep. Nat Neurosci, 19(7), 959-964. https://doi.org/10.1038/nn.4304

Malerba, P., Jones, M. W., \& Bazhenov, M. A. (2017). "Defining the synaptic mechanisms that tune CA3-CA1 reactivation during sharp-wave ripples". bioRxiv, 164699. https://doi.org/10.1101/164699 
Maviel, T., Durkin, T. P., Menzaghi, F., \& Bontempi, B. (2004). Sites of neocortical reorganization critical for remote spatial memory [Research Support, Non-U.S. Gov't]. Science, 305(5680), 96-99. https://doi.org/10.1126/science.1098180

McAvoy, K. M., \& Sahay, A. (2017). Targeting Adult Neurogenesis to Optimize Hippocampal Circuits in Aging. Neurotherapeutics, 14(3), 630-645. https://doi.org/10.1007/s13311-017-0539-6

McClelland, J. L., McNaughton, B. L., \& O'Reilly, R. C. (1995). Why there are complementary learning systems in the hippocampus and neocortex: insights from the successes and failures of connectionist models of learning and memory. Psychol Rev, 102(3), 419-457.

McKenzie, S., Frank, A. J., Kinsky, N. R., Porter, B., Rivière, P. D., \& Eichenbaum, H. (2014). Hippocampal representation of related and opposing memories develop within distinct, hierarchically organized neural schemas. Neuron, 83(1), 202-215. https://doi.org/10.1016/j.neuron.2014.05.019

McKenzie, S., Robinson, N. T., Herrera, L., Churchill, J. C., \& Eichenbaum, H. (2013). Learning causes reorganization of neuronal firing patterns to represent related experiences within a hippocampal schema. J Neurosci, 33(25), 10243-10256. https://doi.org/10.1523/JNEUROSCI.0879-13.2013

Modi, M. N., Dhawale, A. K., \& Bhalla, U. S. (2014). CA1 cell activity sequences emerge after reorganization of network correlation structure during associative learning. Elife, 3, e01982. https://doi.org/10.7554/eLife.01982

Mori, M., Gahwiler, B. H., \& Gerber, U. (2007). Recruitment of an inhibitory hippocampal network after bursting in a single granule cell [Research Support, Non-U.S. Gov't]. Proc Natl Acad Sci U S A, 104(18), 7640-7645. https://doi.org/10.1073/pnas.0702164104

Morrissey, M. D., Insel, N., \& Takehara-Nishiuchi, K. (2017). Generalizable knowledge outweighs incidental details in prefrontal ensemble code over time. Elife, 6. https://doi.org/10.7554/eLife.22177

Nadel, L., \& Moscovitch, M. (1997). Memory consolidation, retrograde amnesia and the hippocampal complex. Curr Opin Neurobiol, 7(2), 217-227. https://doi.org/10.1016/s0959-4388(97)80010-4

Nakashiba, T., Buhl, D. L., McHugh, T. J., \& Tonegawa, S. (2009). Hippocampal CA3 output is crucial for ripple-associated reactivation and consolidation of memory. Neuron, 62(6), 781-787. https://doi.org/10.1016/j.neuron.2009.05.013

Neubrandt, M., Olah, V. J., Brunner, J., \& Szabadics, J. (2017). Feedforward inhibition is randomly wired from individual granule cells onto CA3 pyramidal cells. Hippocampus, 27(10), 1034-1039. https://doi.org/10.1002/hipo.22763

Ognjanovski, N., Schaeffer, S., Wu, J., Mofakham, S., Maruyama, D., Zochowski, M., \& Aton, S. J. (2017). Parvalbuminexpressing interneurons coordinate hippocampal network dynamics required for memory consolidation. Nat Commun, 8, 15039. https://doi.org/10.1038/ncomms15039

Pan, S., Mayoral, S. R., Choi, H. S., Chan, J. R., \& Kheirbek, M. A. (2020). Preservation of a remote fear memory requires new myelin formation. Nat Neurosci, 23(4), 487-499. https://doi.org/10.1038/s41593-019-0582-1

Peyrache, A., Khamassi, M., Benchenane, K., Wiener, S. I., \& Battaglia, F. P. (2009). Replay of rule-learning related neural patterns in the prefrontal cortex during sleep. Nat Neurosci, 12(7), 919-926. https://doi.org/10.1038/nn.2337

Rajasethupathy, P., Sankaran, S., Marshel, J. H., Kim, C. K., Ferenczi, E., Lee, S. Y., Berndt, A., Ramakrishnan, C., Jaffe, A., Lo, M., Liston, C., \& Deisseroth, K. (2015). Projections from neocortex mediate top-down control of memory retrieval. Nature, 526(7575), 653-659. https://doi.org/10.1038/nature15389

Rubin, A., Geva, N., Sheintuch, L., \& Ziv, Y. (2015). Hippocampal ensemble dynamics timestamp events in long-term memory. Elife, 4. https://doi.org/10.7554/eLife.12247

Ruediger, S., Spirig, D., Donato, F., \& Caroni, P. (2012). Goal-oriented searching mediated by ventral hippocampus early in trial-and-error learning. Nat Neurosci, 15(11), 1563-1571. https://doi.org/10.1038/nn.3224

Ruediger, S., Vittori, C., Bednarek, E., Genoud, C., Strata, P., Sacchetti, B., \& Caroni, P. (2011). Learning-related feedforward inhibitory connectivity growth required for memory precision. Nature, 473(7348), 514-518. https://doi.org/10.1038/nature09946

Sasaki, T., Piatti, V. C., Hwaun, E., Ahmadi, S., Lisman, J. E., Leutgeb, S., \& Leutgeb, J. K. (2018). Dentate network activity is necessary for spatial working memory by supporting CA3 sharp-wave ripple generation and prospective firing of CA3 neurons. Nat Neurosci, 21(2), 258-269. https://doi.org/10.1038/s41593-017-0061-5 
Schapiro, A. C., Turk-Browne, N. B., Botvinick, M. M., \& Norman, K. A. (2017). Complementary learning systems within the hippocampus: a neural network modelling approach to reconciling episodic memory with statistical learning. Philos Trans R Soc Lond B Biol Sci, 372(1711). https://doi.org/10.1098/rstb.2016.0049

Sheintuch, L., Rubin, A., Brande-Eilat, N., Geva, N., Sadeh, N., Pinchasof, O., \& Ziv, Y. (2017). Tracking the Same Neurons across Multiple Days in Ca. Cell Rep, 21(4), 1102-1115. https://doi.org/10.1016/j.celrep.2017.10.013

Soltesz, I., \& Losonczy, A. (2018). CA1 pyramidal cell diversity enabling parallel information processing in the hippocampus. Nat Neurosci, 21(4), 484-493. https://doi.org/10.1038/s41593-018-0118-0

Squire, L. R. (2004). Memory systems of the brain: a brief history and current perspective. Neurobiol Learn Mem, 82(3), 171-177. https://doi.org/10.1016/i.nlm.2004.06.005

Stark, E., Roux, L., Eichler, R., Senzai, Y., Royer, S., \& Buzsaki, G. (2014). Pyramidal cell-interneuron interactions underlie hippocampal ripple oscillations. Neuron, 83(2), 467-480. https://doi.org/10.1016/j.neuron.2014.06.023

Steadman, P. E., Xia, F., Ahmed, M., Mocle, A. J., Penning, A. R. A., Geraghty, A. C., Steenland, H. W., Monje, M., Josselyn, S. A., \& Frankland, P. W. (2020). Disruption of Oligodendrogenesis Impairs Memory Consolidation in Adult Mice. Neuron, 105(1), 150-164 e156. https://doi.org/10.1016/j.neuron.2019.10.013

Strange, B. A., Witter, M. P., Lein, E. S., \& Moser, E. I. (2014). Functional organization of the hippocampal longitudinal axis [Review]. Nat Rev Neurosci, 15(10), 655-669. https://doi.org/10.1038/nrn3785

Takehara-Nishiuchi, K., \& McNaughton, B. L. (2008). Spontaneous changes of neocortical code for associative memory during consolidation. Science, 322(5903), 960-963. https://doi.org/10.1126/science.1161299

Teyler, T. J., \& DiScenna, P. (1986). The hippocampal memory indexing theory. Behav Neurosci, 100(2), 147-154.

Tompary, A., \& Davachi, L. (2017). Consolidation Promotes the Emergence of Representational Overlap in the Hippocampus and Medial Prefrontal Cortex. Neuron, 96(1), 228-241 e225.

https://doi.org/10.1016/j.neuron.2017.09.005

Tonegawa, S., Morrissey, M. D., \& Kitamura, T. (2018). The role of engram cells in the systems consolidation of memory. Nat Rev Neurosci, 19(8), 485-498. https://doi.org/10.1038/s41583-018-0031-2

Torborg, C. L., Nakashiba, T., Tonegawa, S., \& McBain, C. J. (2010). Control of CA3 output by feedforward inhibition despite developmental changes in the excitation-inhibition balance . J Neurosci, 30(46), 15628-15637. https://doi.org/10.1523/JNEUROSCl.3099-10.2010

Tse, D., Langston, R. F., Kakeyama, M., Bethus, I., Spooner, P. A., Wood, E. R., Witter, M. P., \& Morris, R. G. (2007). Schemas and memory consolidation. Science, 316(5821), 76-82. https://doi.org/10.1126/science.1135935

Vetere, G., Xia, F., Ramsaran, A. I., Tran, L. M., Josselyn, S. A., \& Frankland, P. W. (2021). An inhibitory hippocampalthalamic pathway modulates remote memory retrieval. Nat Neurosci. https://doi.org/10.1038/s41593-021$\underline{00819-3}$

Wilson, M. A., \& McNaughton, B. L. (1994). Reactivation of hippocampal ensemble memories during sleep. Science, 265(5172), 676-679.

Wiltgen, B. J., Zhou, M., Cai, Y., Balaji, J., Karlsson, M. G., Parivash, S. N., Li, W., \& Silva, A. J. (2010). The hippocampus plays a selective role in the retrieval of detailed contextual memories [Research Support, N.I.H., Extramural]. Curr Biol, 20(15), 1336-1344. https://doi.org/10.1016/j.cub.2010.06.068

Winocur, G., Moscovitch, M., \& Bontempi, B. (2010). Memory formation and long-term retention in humans and animals: convergence towards a transformation account of hippocampal-neocortical interactions. Neuropsychologia, 48(8), 2339-2356. https://doi.org/10.1016/j.neuropsychologia.2010.04.016

Winocur, G., Moscovitch, M., \& Sekeres, M. (2007). Memory consolidation or transformation: context manipulation and hippocampal representations of memory. Nat Neurosci, 10(5), 555-557. https://doi.org/10.1038/nn1880

Xia, F., Richards, B. A., Tran, M. M., Josselyn, S. A., Takehara-Nishiuchi, K., \& Frankland, P. W. (2017). Parvalbuminpositive interneurons mediate neocortical-hippocampal interactions that are necessary for memory consolidation. Elife, 6. https://doi.org/10.7554/eLife.27868

Yiu, A. P., Mercaldo, V., Yan, C., Richards, B., Rashid, A. J., Hsiang, H. L., Pressey, J., Mahadevan, V., Tran, M. M., Kushner, S. A., Woodin, M. A., Frankland, P. W., \& Josselyn, S. A. (2014). Neurons are recruited to a memory trace based on relative neuronal excitability immediately before training. Neuron, 83(3), 722-735. 
bioRxiv preprint doi: https://doi.org/10.1101/2021.05.21.445117; this version posted May 21, 2021. The copyright holder for this preprint (which

was not certified by peer review) is the author/funder, who has granted bioRxiv a license to display the preprint in perpetuity. It is made available under aCC-BY-NC-ND 4.0 International license.

0 Zhou, P., Resendez, S. L., Rodriguez-Romaguera, J., Jimenez, J. C., Neufeld, S. Q., Giovannucci, A., Friedrich, J., Pnevmatikakis, E. A., Stuber, G. D., Hen, R., Kheirbek, M. A., Sabatini, B. L., Kass, R. E., \& Paninski, L. (2018). Efficient and accurate extraction of in vivo calcium signals from microendoscopic video data. Elife, 7. https://doi.org/10.7554/eLife.28728

Zhou, Y., Won, J., Karlsson, M. G., Zhou, M., Rogerson, T., Balaji, J., Neve, R., Poirazi, P., \& Silva, A. J. (2009). CREB regulates excitability and the allocation of memory to subsets of neurons in the amygdala. Nat Neurosci, 12(11), 1438-1443. https://doi.org/10.1038/nn.2405 
bioRxiv preprint doi: https://doi.org/10.1101/2021.05.21.445117; this version posted May 21, 2021. The copyright holder for this preprint (which was not certified by peer review) is the author/funder, who has granted bioRxiv a license to display the preprint in perpetuity. It is made available under aCC-BY-NC-ND 4.0 International license.

a

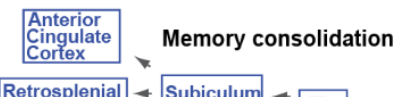

Retrosplenial
Cortex Subiculum 4 CA1

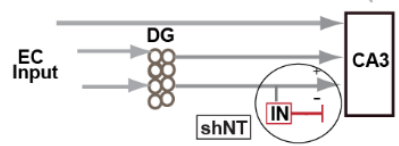

MFT

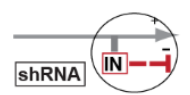

C

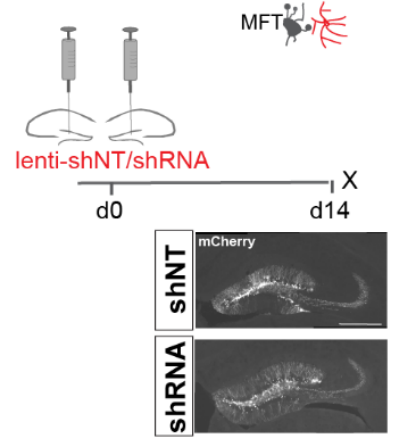

d

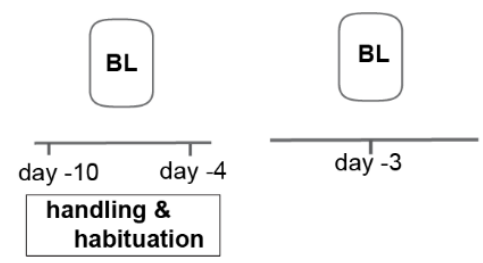

e

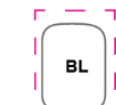

$$
+\underset{\text { day }-3\lrcorner}{+}
$$

baseline imaging f
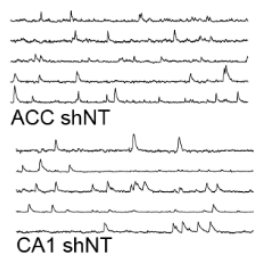

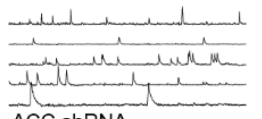
ACC shRNA

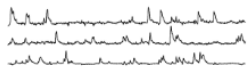
of b

Figure 1
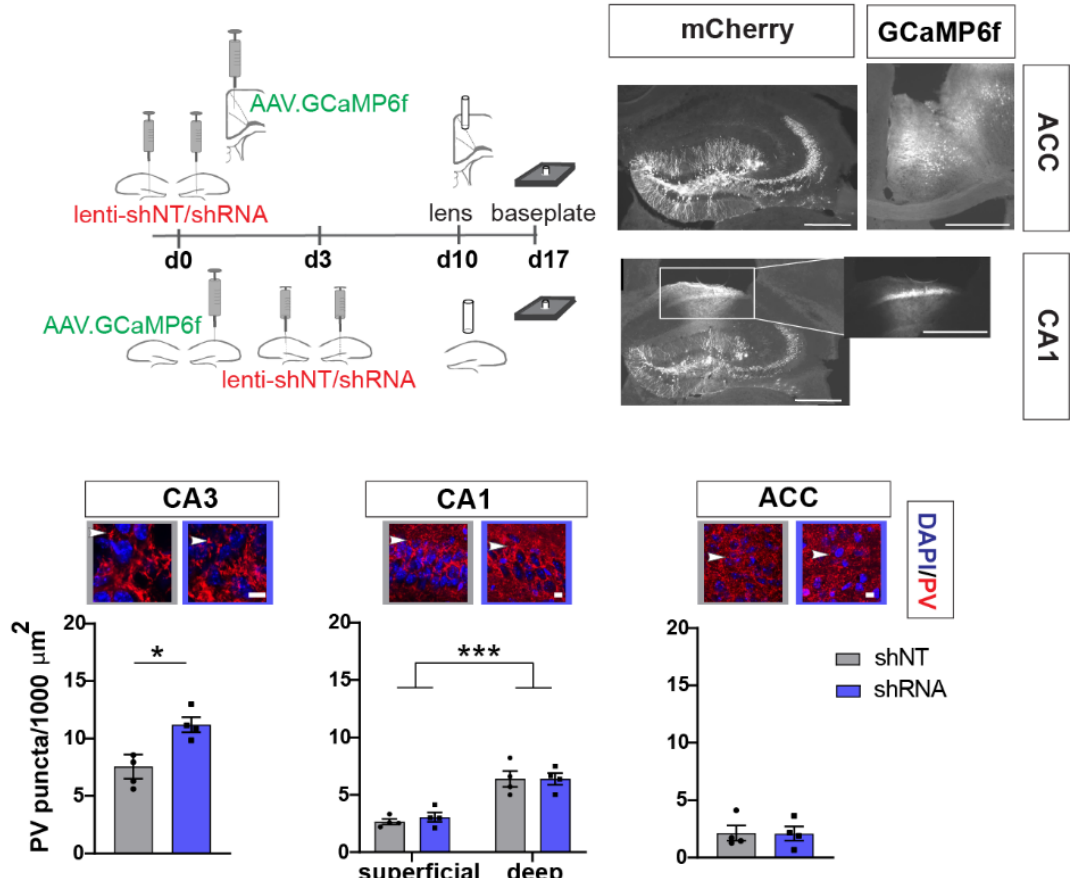

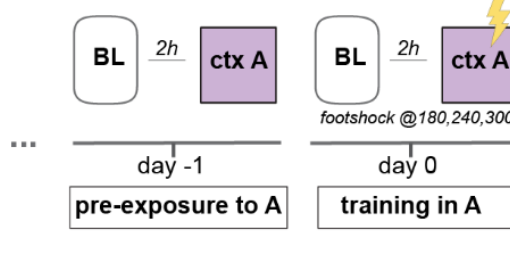

g
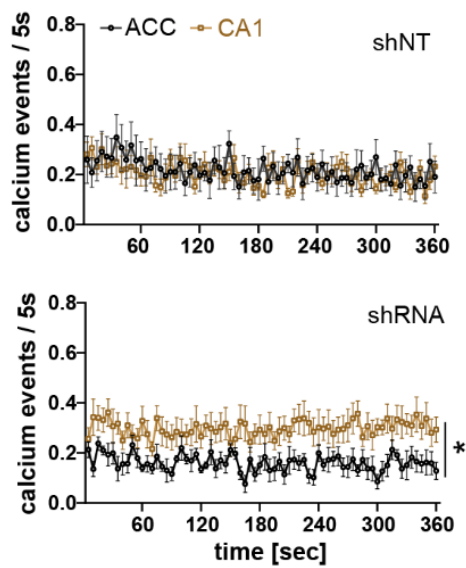

h
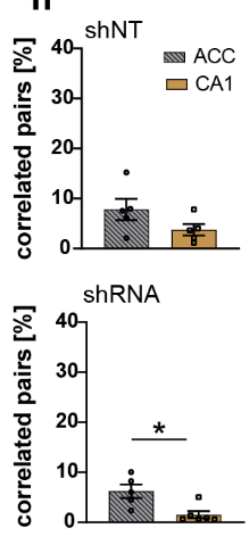

i
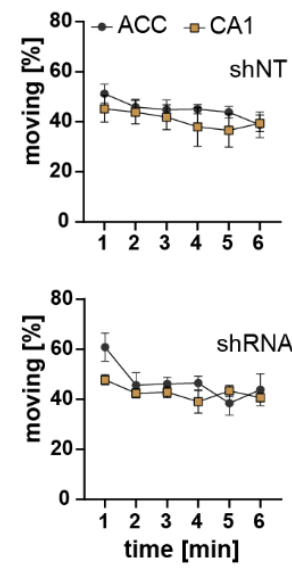


\section{Figure 1: Cross region comparisons in learning-naïve mice following enhancement of FFI in DG-CA3}

a) Simplified wiring diagram of hippocampal-cortical circuits supporting memory consolidation. Highlighted is the DG-PV IN-CA3 inhibitory microcircuit. Dentate granule cell projections (Mossy fibers, MF) synapse onto pyramidal neurons in CA3 and also release glutamate directly onto stratum lucidum PV-positive interneurons (PV INs, red) through filopodia that emanate from mossy fiber terminals (MFTs, grey). Injection of lenti-shRNA into dentate gyrus (DG) increased MFT filopodia and dentate granule cell recruitment of PV INs. In response to increased DGC excitatory drive PV INs elaborate inhibitory synaptic contacts in CA3 and feed-forward inhibition onto CA3 neurons (feed-forward inhibition, FFI). Pyramidal neurons in CA3 project to CA1 that sends projections to multiple brain regions such as the anterior cingulate cortex (ACC). b) Schematic workflow and example of virus injection and lens implantation. Mice received unilateral injection of AAV1.CaMKII.GCaMP6f.WPRES.SV40 (GCaMP6f, left panel) in the region of interest and bilateral injection of lenti-shNT or lenti-shRNA virus (mCherry, right panel) in the hilus of dorsal DG. In CA1 implanted mice, the injections were separated by 3 days to allow for best targeting and expression. Scale bar: $500 \mu$ m. c) Quantification and representative images of PV puncta (white arrows) in CA3 (left), the deeper and superficial layer of CA1 (middle) and ACC (right) per ROI (1000 $\mu^{2}$, scale bar: $\left.10 \mu \mathrm{m}\right)$ in behaviorally naïve mice 2 weeks post injection (left panel, scale bar: $500 \mu \mathrm{m}$ ). Images show representative examples of ROIs from shNT (left) and shRNA (right) injected mice. Arrows indicate a PV puncta example. Bar graphs represent mean +/- SEM. Scatter represent individual mice. Injection of lenti-shRNA significantly increased PV puncta in CA3 [Two-tailed Mann-Whitney: $\mathrm{p}=0.029, \mathrm{n}=4$ ] but not in CA1 [Two-way repeated measures ANOVA, layer $\mathrm{x}$ virus effect $\mathrm{p}=0.583$ : virus main effect $\mathrm{p}=0.752$, layer main effect: $\mathrm{p}<0.0001$ ]; or ACC [Two-tailed Mann-Whitney test: $\mathrm{p}=0.886, \mathrm{n}=4$ ]. In CA1, a significant difference was found between layers. d) Sketch of behavior paradigm. Prior to behavioral testing and calcium imaging, mice were handled and habituated to the microscope and baseline (BL) environment. Each recording day began with a BL recording followed by a test with at least $2 \mathrm{~h}$ between each session. On days with multiple test sessions (recall), the order of context (context A or context $\mathrm{C}$ ) was counterbalanced. BL=Baseline, ctx $\mathrm{A}=$ context $\mathrm{A}$, ctx $\mathrm{C}=$ context $\mathrm{C}$. e) Schematic of time point in behavioral paradigm of the presented data (BL on first recording day).f) Example raw traces of changes is calcium extracted with CNMF-E. Each line represents one neuron in the field of view. g) Comparison of calcium events (averaged over 5 second time bins) in ACC and CA1 in shNT (upper) and shRNA (lower) mice (mean +/- SEM, $\mathrm{n}=$ number of mice) [Two-way repeated measures ANOVA, brain region $x$ time effect $p<0.0001$; brain region main effect $p=0.833$, time main effect $p=0.026, A C C$ $\mathrm{n}=5$, CA1 $\mathrm{n}=5$ ]. In shRNA mice, we saw significantly more calcium events in CA1 compared to ACC [Two-way repeated measures ANOVA, brain region $x$ time effect $p<0.0001$; brain region main effect $p=0.046$, time main effect $p=0.134$, ACC $n=5$, CA1 $n=6]$. h) Number of correlated pairs (correlation $>$ mean+2SD from shuffled data) expressed as percentage of all possible pairs. In lenti-shRNA injected mice the number of correlated pairs in CA1 was significantly lower compared to ACC [unpaired t-test with Welch's correction, shNT: ACC vs CA1 $\mathrm{p}=0.144, \mathrm{n}=5$; shRNA: ACC vs CA1: $\mathrm{p}=0.0212, \mathrm{n}=5$ ACC, $\mathrm{n}=6$ CA1). i) Quantification of the time mice were moving during the recording. We found no difference between experimental groups [Two-way repeated measures ANOVA, shNT, upper panel: time $x$ brain region effect $p=0.7199$, time main effect: $p=0.072$, brain region main effect: $p=0.463, n=5$; shRNA lower panel: time $x$ brain region effect: $p=0.213$, time main effect: $p=0.025$, brain region main effect $\mathrm{p}=0.21, \mathrm{ACC} \mathrm{n}=5, \mathrm{CA} 1 \mathrm{n}=6] .{ }^{*} \mathrm{p}<0.05, * * \mathrm{p}<0.01, * * * \mathrm{p}<0.001$. 
bioRxiv preprint doi: https://doi.org/10.1101/2021.05.21.445117; this version posted May 21, 2021. The copyright holder for this preprint (which

was not certified by peer review) is the author/funder, who has granted bioRxiv a license to display the preprint in perpetuity. It is made available under aCC-BY-NC-ND 4.0 International license.

Figure 2

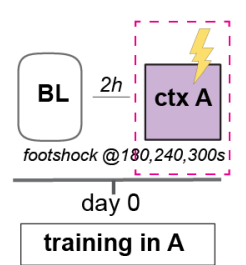

b

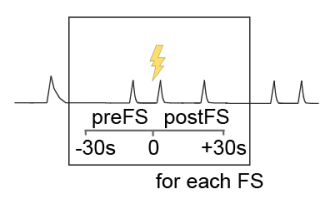

d

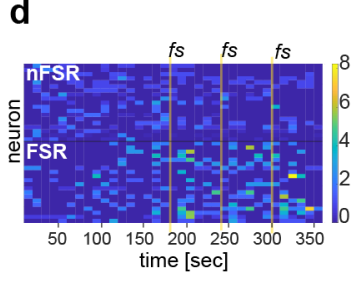

f

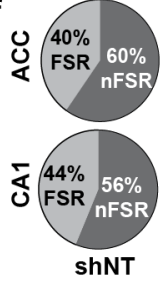

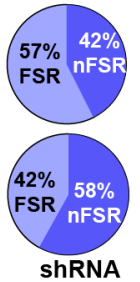

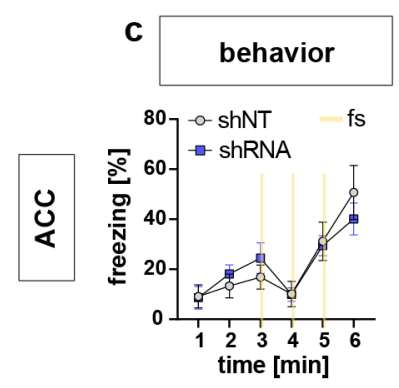

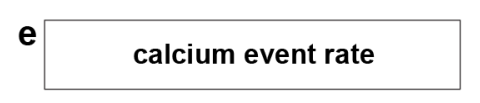
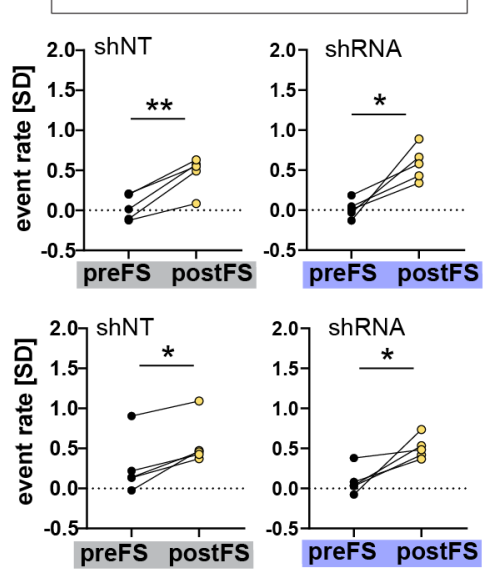

$\mathbf{h}$

correlated pairs
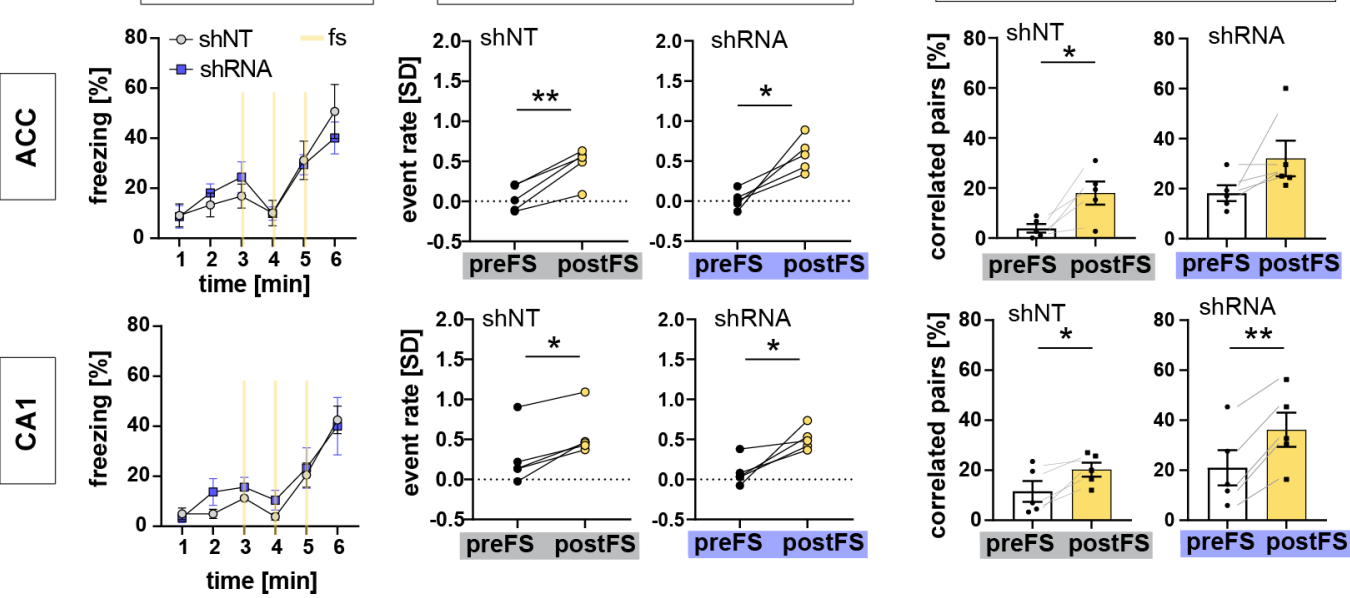

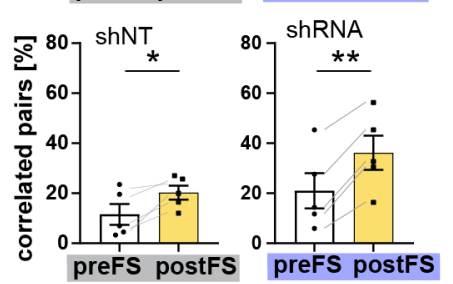

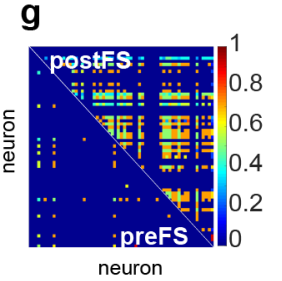

i shNT
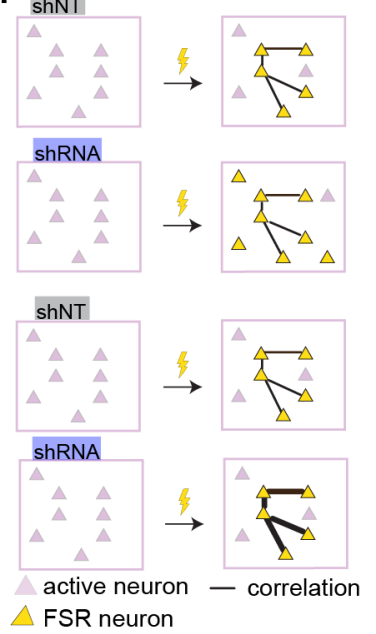


\section{$9 \quad$ Figure 2: Increasing FFI promotes neuronal responses in CA1 and ACC during encoding of foot-shock}

0 a) Schematic of time point in behavioral paradigm of the presented data (context A on training day with 3 foot1 shocks (FS) applied at 180, 240 and 360 seconds). b) schematic of analysis window for neuronal activity before 2 FS (preFS) and after FS (postFS) presented in panel e,g and h.c) Quantification of FS response behavior measured 3 as freezing in percentage of one minute time bins. All experimental groups showed a similar increase in freezing 4 upon the FS training in context A with no main treatment effect [upper panel, ACC: Two-way repeated measures 5 ANOVA, time $x$ treatment effect $\mathrm{p}=0.702$ : time main effect $\mathrm{p}<0.0001$, treatment main effect $=0.796$, $\mathrm{n}=5$; lower 6 panel CA1: time $x$ treatment effect $\mathrm{p}=0.838$, time main effect $\mathrm{p}<0.0001$, treatment main effect $=0.49, \mathrm{n}=5 \mathrm{shNT}$, $7 \mathrm{n}=6$ shRNA]. d) Example heatmap of z-scored neuronal activity (calcium events) in CA1 (shNT) across time in 8 context A grouped by neuronal activity (foot-shock responsive, FSR; non-foot-shock responsive, nFSR). Colorbar 9 represents SD (z-scored to first $180 \mathrm{sec}$ of the recording). e) Neuronal activity of FSR neurons increased 0 significantly after the FS was applied in all experimental groups. Neuronal activity was z-scored (over the first $1180 \mathrm{sec}$ in context A) and averaged over the 3 foot-shocks using a 30sec window as preFS or postFS condition 2 (see sketch in panel b) [Two-tailed paired t-test, ACC shNT preFS vs postFS: $\mathrm{p}=0.004$ (upper left); ACC shRNA 3 preFS vs postFS: $\mathrm{p}=0.013$ (upper right), CA1 shNT preFS vs postFS: $\mathrm{p}=0.007$ (lower left), CA1 shRNA preFS 4 vs postFS: $\mathrm{p}=0.024$ (lower right), $\mathrm{n}=5$ ].f) Number of FSR and $\mathrm{nFSR}$ neurons in each experimental group In ACC, 5 lenti-shRNA injection increased the number of FSR neurons (see Fig2-suppl 1 for statistical analysis). g) Example 6 heatmap of pairwise correlation between neuronal pairs in CA1 before the FS (preFS, lower triangle) and after 7 the FS (postFS, upper triangle) from one shNT injected mouse.). Colorbar represents correlation coefficient $\mathbf{h}$ ) 8 Number of correlated pairs ( >mean+2SD from a shuffled control) among FSR neurons as percentage of all 9 possible pairs of FSR neurons. All experimental groups, except ACC shRNA, showed an increase in the 0 percentage of correlated pairs after the foot-shock. [Two-tailed paired t-test, ACC shNT: $\mathrm{p}=0.021$ (upper left), 1 ACC shRNA: $\mathrm{p}=0.141$ (upper right), CA1 shNT: $\mathrm{p}=0.021$ (lower left), CA1 shRNA: $\mathrm{p}=0.001$ (lower right), $\mathrm{n}=$ 2 5]. i) Graphic summary of findings presented in this panel. In both ACC and CA1, learning, in form of 3 experiencing a foot-shock in context A, induced the formation of a neural ensemble among FSR neurons by 4 increasing co-activity. Increased FFI facilitated this effect in both brain regions with a higher number of FSR 5 neurons in ACC and a stronger increase in correlated pairs in CA1 (see also Fig2-suppl 1). Throughout, statistical 6 data are represented as mean \pm SEM, $* \mathrm{p}<0.05, * * \mathrm{p}<0.01, * * * \mathrm{p}<0.001$. 
bioRxiv preprint doi: https://doi.org/10.1101/2021.05.21.445117; this version posted May 21, 2021. The copyright holder for this preprint (which

was not certified by peer review) is the author/funder, who has granted bioRxiv a license to display the preprint in perpetuity. It is made available under aCC-BY-NC-ND 4.0 International license.

\section{Suppl Figure 1}
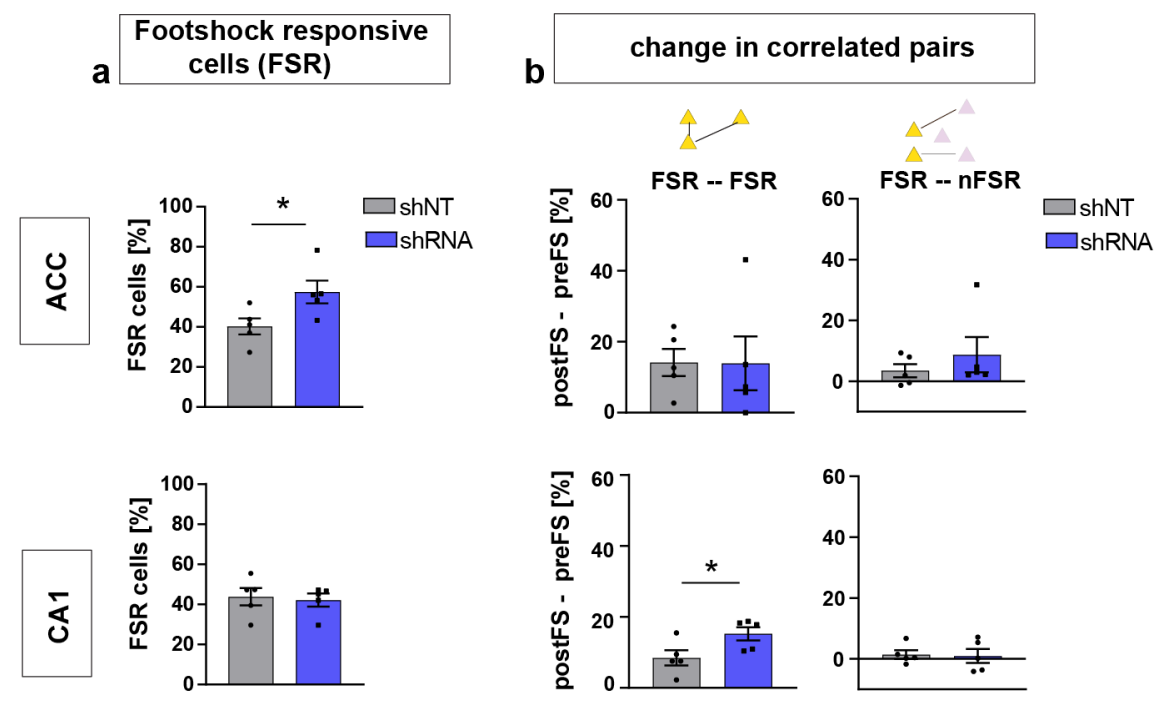


\section{Supplementary Figure 1: Supporting data for Figure 2}

9 a) Quantification of FSR neurons in ACC (upper panel) and CA1 (lower panel). In ACC, shRNA mice showed 0 significantly more FSR compared to shNT mice [Two-tailed unpaired t test with Welch's correction, $\mathrm{p}=0.043$, $1 \mathrm{n}=5$ ] while there was no difference in CA1 [Two-tailed unpaired t test with Welch's correction $\mathrm{p}=0.743, \mathrm{n}=5$ ] $\mathbf{b}$ ) 2 Change in correlated pairs [postFS - preFS] within FSR neurons (left panels) and across FSR and nFSR neurons. 3 In CA1, the increase of correlated pairs within FSR neurons was significantly higher in shRNA mice compared 4 to shNT mice [Two-tailed unpaired t test with Welch's correction, $\mathrm{p}=0.044, \mathrm{n}=5$ ] while there was no difference 5 in ACC [Two-tailed unpaired t test with Welch's correction $\mathrm{p}=0.980, \mathrm{n}=5$ ]. In both brain regions, there is no 6 change in correlation between pairs of FSR and nFSR neurons (upper and lower right panel) [One-sample t-test 7 against 0; ACC shNT: p=0.186 ACC shRNA: p=0.204 (upper right), CA1 shNT: p=0.382, CA1 shRNA: $8 \mathrm{p}=0.698$, (lower right), $\mathrm{n}=5]$. 
a
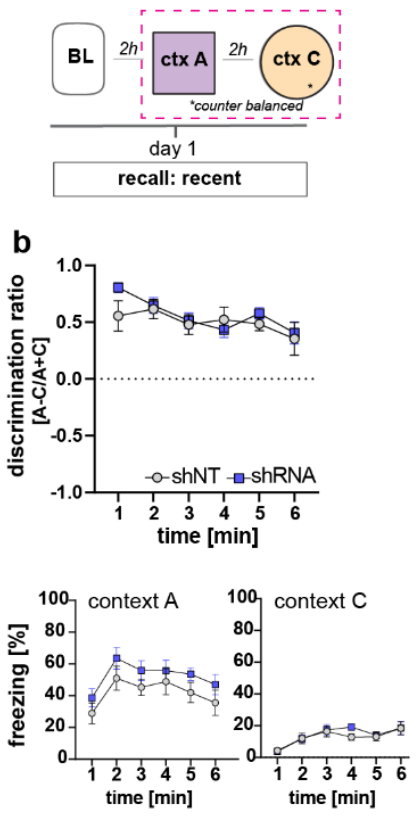

i
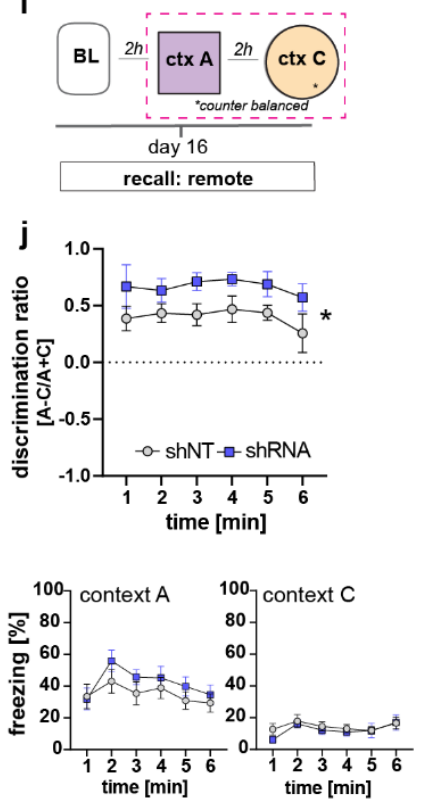

c
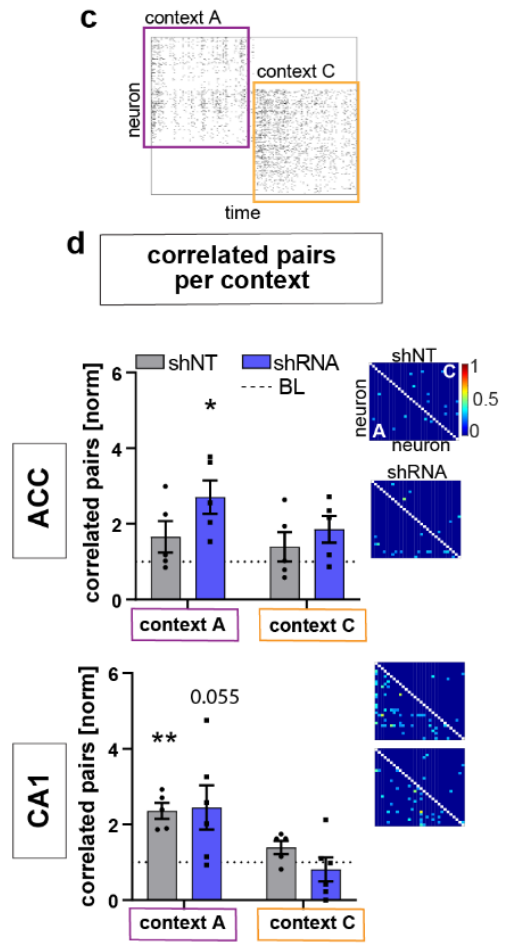

$\mathbf{k}$
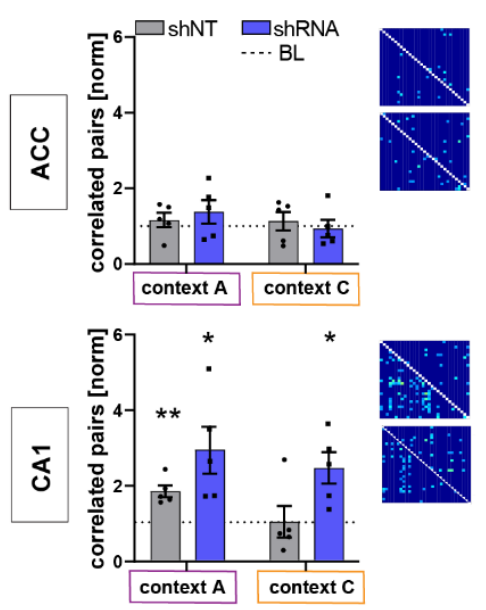

e
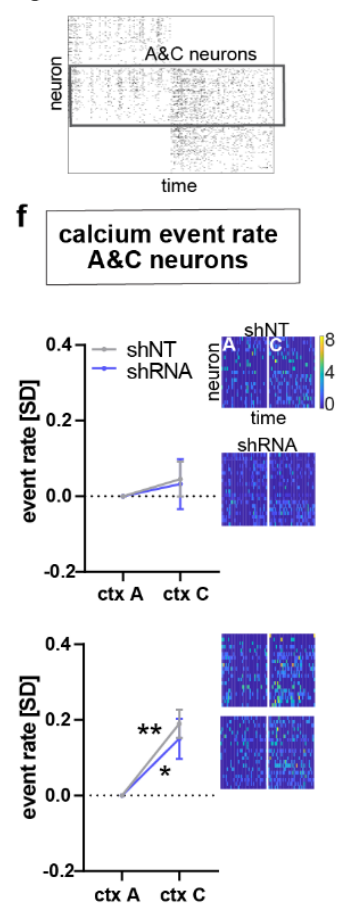

I
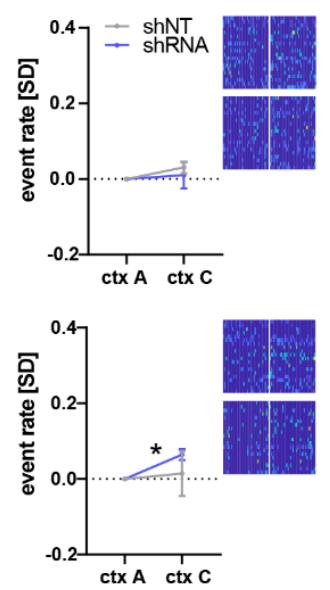

g
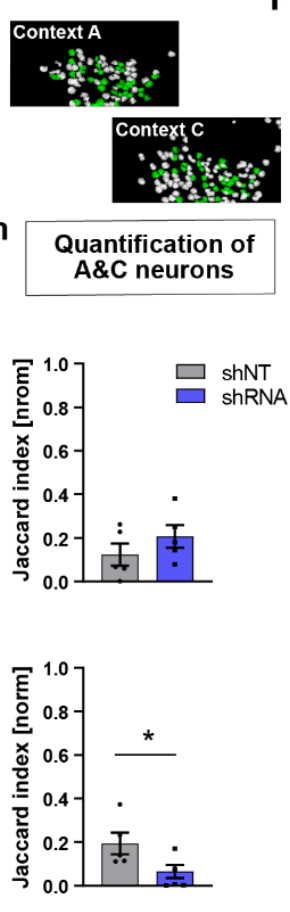

Figure 3
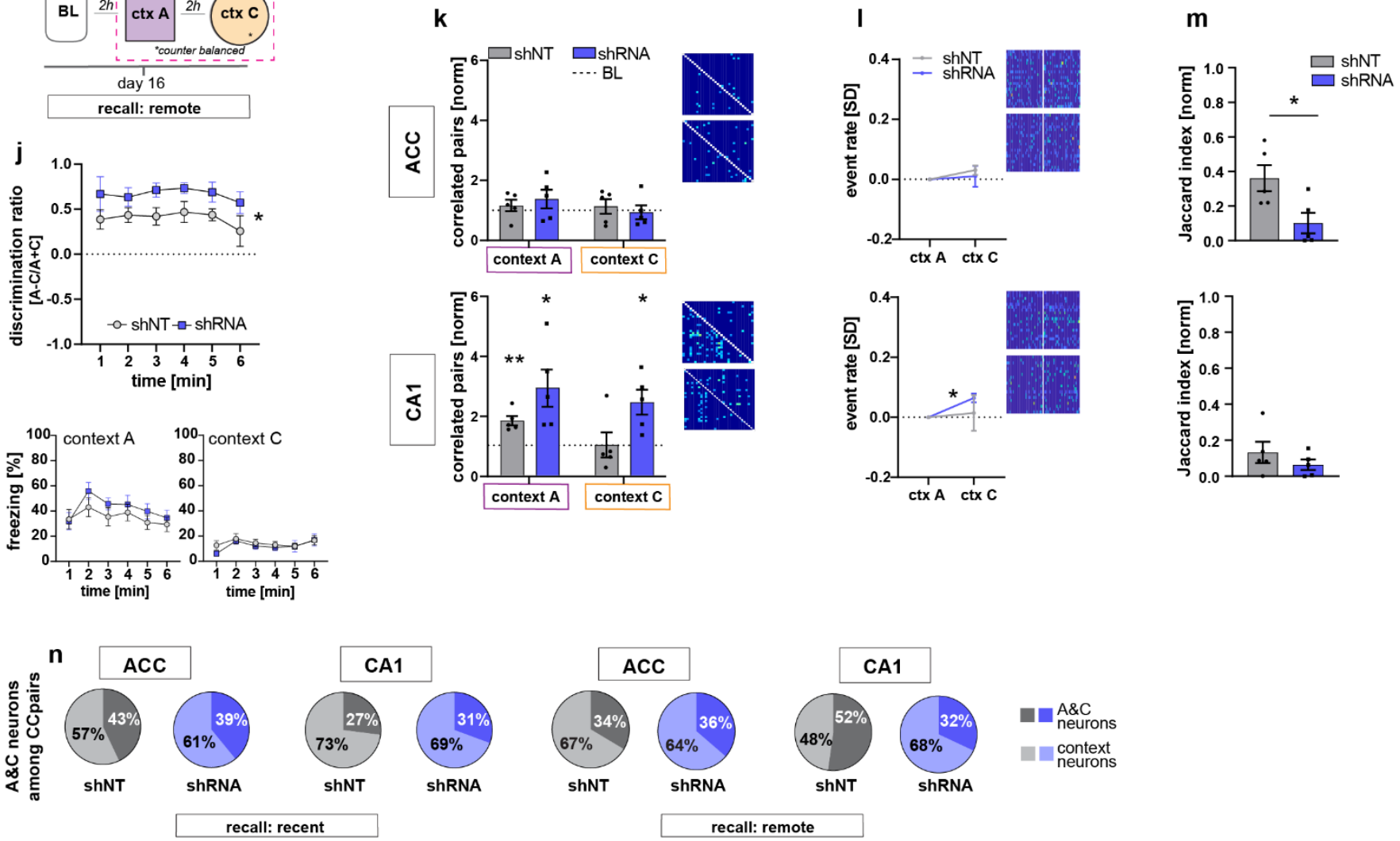
Figure 3: FFI in DG-CA3 dictates strength and specificity of CA1-ACC ensembles during memory consolidation. a) Schematic of time point in behavioral paradigm of the presented data (context A and context $C$ on day 1 after foot-shock training in context A). b) Quantification of discrimination ratio as relative difference in freezing in context $\mathrm{A}$ and context $\mathrm{C}$ (upper panel) and freezing time in percentage of one minute time bins in context A (lower left) and context C (lower right). ACC and CA1 mice were grouped together by virus. One day after training in context $\mathrm{A}$, all mice discriminated between context $\mathrm{A}$ and $\mathrm{C}$ with no difference between treatment groups [Two-way repeated measures ANOVA, treatment $\mathrm{x}$ time effect $\mathrm{p}=0.434$, time main effect $\mathrm{p}=0.014$; treatment main effect $\mathrm{p}=0.232 ; \mathrm{n}=10$, pooled $\mathrm{CA} 1$ and $\mathrm{ACC}$ mice]. c) Raster plot of calcium events over time in context $\mathrm{A}$ and context $\mathrm{C}$ after registering neurons across sessions. Each row corresponds to a neuron, $\mathrm{x}$-axis corresponds to time (360 seconds) in each context. Colored squares indicate the context the recording was acquired in. Empty rows in one context mean that the neuron was not active in this context e.g. top rows neuron are only active in context A but not in context C. d) Quantification of number of correlated pairs across all neurons per context. Context-specific neuronal pairs were analyzed by normalizing the correlated pairs to the baseline level recorded in the same day baseline recording (dotted line in graph). Heatmaps right to the graph show correlation values of example neurons in context A (lower triangle) and context C (upper triangle). In each graph, the upper heatmap shows an example of a lenti-shNT injected, the lower heatmap of a lenti-shRNA injected mouse. In CA1, both lenti-shNT and lenti-shRNA injected mice showed a higher number of correlated pairs compared to baseline in context $\mathrm{A}$ but not in the new context $\mathrm{C}$ [one sample $\mathrm{t}$ test against 1, context $\mathrm{A}$, shNT: $\mathrm{p}=0.003$, shRNA: $\mathrm{p}=0.055$, context $\mathrm{C}: \mathrm{shNT}: \mathrm{p}=0.089$, shRNA: $\mathrm{p}=0.574, \mathrm{n}=5]$. In ACC, only lenti-shRNA injected mice showed a significantly higher number of correlated pairs in context A compared to baseline [one sample $t$ test against 1 , context $\mathrm{A}$, shNT: $\mathrm{p}=0.188$, shRNA: $\mathrm{p}=0.018$; context $\mathrm{C}$ : shNT: $\mathrm{p}=0.368$, shRNA: $\mathrm{p}=0.073$, $\mathrm{n}=5$ ]. e) Raster plot of calcium events over time in context $\mathrm{A}$ and context $\mathrm{C}$ after registering neurons across sessions (same as in panel c). Each row corresponds to a neuron. The square marks neurons that were active in both contexts (A\&C neurons). f) Normalized activity of A\&C neurons. Heatmaps show activity of example neurons in context A (left side) and context $\mathrm{C}$ (right side) in the first $180 \mathrm{sec}$ per context for better visualization. In each graph, the upper heatmap shows an example of a lenti-shNT injected, the lower heatmap of a lenti-shRNA injected mouse. A\&C neurons showed a significantly higher activity in context $\mathrm{C}$ compared to context $\mathrm{A}$ in $\mathrm{CA} 1$ but not in ACC [one sample t test against 0 , ACC, shNT: $\mathrm{p}=0.399$, shRNA: $\mathrm{p}=0.65$; CA1, shNT: $\mathrm{p}=0.007$, shRNA: $\mathrm{p}=0.048, \mathrm{n}=5$ ] . g) Example spatial maps of neurons (white circles) in context $\mathrm{A}$ and context $\mathrm{C}$ with green circles indicating neurons that were registered to be active in both sessions (A\&C neurons). h) Quantification of A\&C neurons using normalized jaccard similarity index. In CA1, lenti-shRNA injected mice showed significantly less $\mathrm{A} \& \mathrm{C}$ neurons compared to lenti-shNT injected mice [Two-tailed unpaired $t$ test with Welch's correction, $\mathrm{p}=$ $0.048, n=5$ ]. No difference was found in ACC [Two-tailed unpaired $t$ test with Welch's correction, $p=0.284$, $\mathrm{n}=5$ ]. i) Schematic of time point in behavioral paradigm of the presented data (context $\mathrm{A}$ and context $\mathrm{C}$ on day 16 after foot-shock training in context A). j) Quantification of discrimination ratio as relative difference in freezing in context $\mathrm{A}$ and context $\mathrm{C}$ (upper panel) and freezing time in percentage of one minute time bins in context $\mathrm{A}$ (lower left) and context $\mathrm{C}$ (lower right panel). ACC and CA1 mice are grouped together by virus. 16 days after foot-shock training in context A, both treatment groups discriminated between A and C. Lenti-shRNA injected mice showed a significantly higher discrimination ratio compared to lenti-shNT injected mice [Two-way repeated measures ANOVA, treatment $\mathrm{x}$ time effect $\mathrm{p}=0.993$, time main effect $\mathrm{p}=0.412$, treatment main effect: $\mathrm{p}=0.028$, $\mathrm{n}=10$, pooled CA1 and ACC mice]. $\mathbf{k}$ ) Quantification of number of correlated pairs across all neurons at day 16. Same presentation as in panel d. In CA1, both virus groups maintained an increased number of correlated neurons 
2 in context A compared to baseline [One-sample t test against 1, context A, shNT, p= 0.006; shRNA, p=0.04; $3 \mathrm{n}=5$ ]. Lenti-shNT mice showed a significantly lower number of correlated pairs compared to day 1 [comparison 4 not shown, Two-tailed paired t-test with Welch's correction, $\mathrm{p}=0.041, \mathrm{n}=5$ ]. In context $\mathrm{C}$, shRNA mice showed 5 a similarly increased number of correlated neurons compared to baseline as in context A [One-sample $t$ test against 61 , context $\mathrm{C}, \mathrm{shNT}, \mathrm{p}=0.973$; shRNA, $\mathrm{p}=0.026$; $\mathrm{n}=5$ ]. In ACC, no difference was found between contexts [One7 sample t test against 1 , context $\mathrm{A}, \mathrm{shNT}, \mathrm{p}=0.444$; shRNA, $\mathrm{p}=0.285$; context $\mathrm{C}, \mathrm{shNT}, \mathrm{p}=0.609$, shRNA, $\mathrm{p}=0.803$, $8 \mathrm{n}=5$ ]. l) Normalized activity of A\&C neurons. Same presentation as in panel $\mathrm{f}$. In CA1, A\&C\& neurons showed 9 a significantly higher activity in context $\mathrm{C}$ compared to context $\mathrm{A}$ in shRNA mice but not in shNT mice [one 0 sample $t$ test against 0 , shNT: $\mathrm{p}=0.824$, shRNA: $\mathrm{p}=0.012, \mathrm{n}=5$ ]. No difference was found in ACC [one sample $\mathrm{t}$ 1 test against $0, \operatorname{shNT}: \mathrm{p}=0.148$, shRNA: $\mathrm{p}=0.784, \mathrm{n}=5$ ]. m) Quantification of A\&C neurons using normalized 2 jaccard similarity index 16 days after foot-shock training. In ACC, lenti-shRNA injected mice showed 3 significantly less A\&C neurons compared to lenti-shNT injected mice [two-tailed unpaired t test with Welch's 4 correction, $\mathrm{p}=0.028, \mathrm{n}=5$ ]. No difference was found in CA1 [two tailed unpaired t test with Welch's correction, $5 \mathrm{p}=0.340, \mathrm{n}=5]$. n) Quantification of A\&C neurons that formed correlated pairs with any other neurons in shNT 6 (grey graphs) and shRNA (blue graphs) injected mice during recent (left panel) and remote (right panel) recall. 7 In CA1, lenti-shRNA injected mice showed fewer A\&C neurons among the correlated pairs compared to lenti8 shNT injected mice during remote recall (see Figure3-suppl1 for statistical analysis). 


\section{suppl Figure 2}

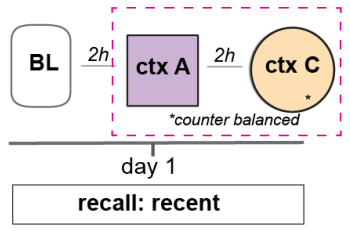

b ACC
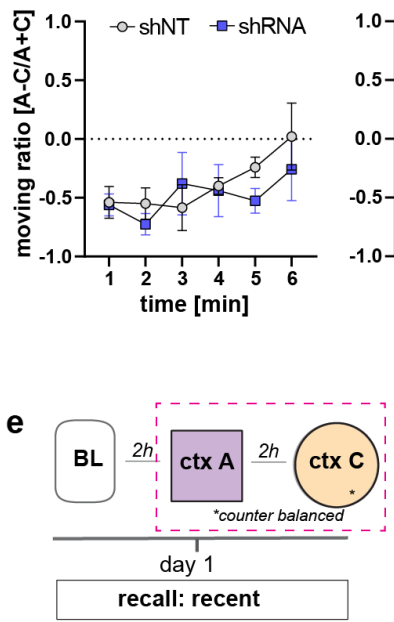

f

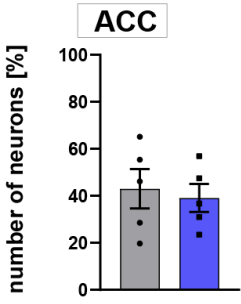

A\&C neurons among CCpairs
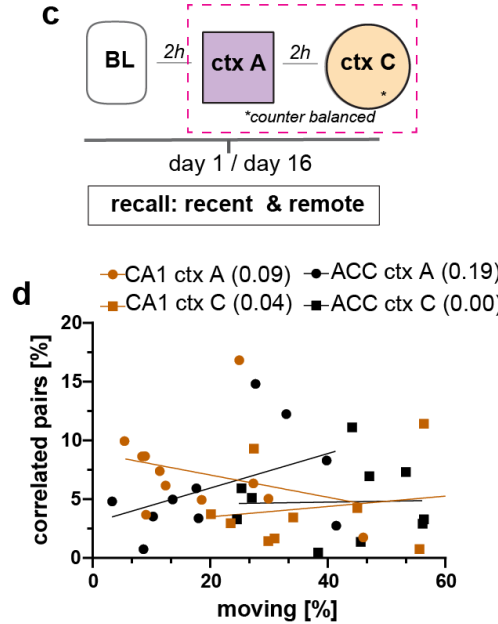

g

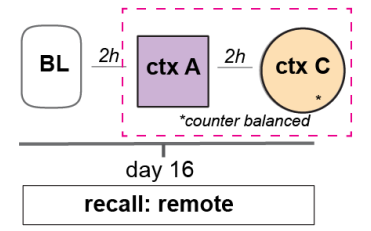

h
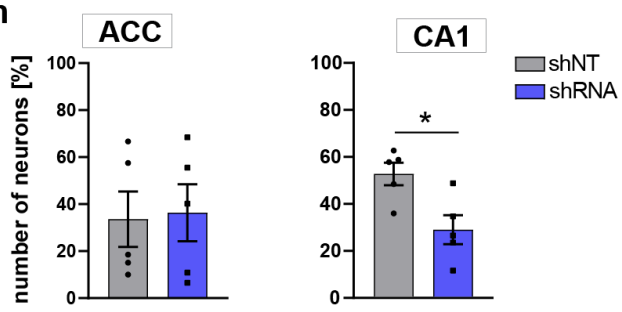

A\&C neurons among CCpairs
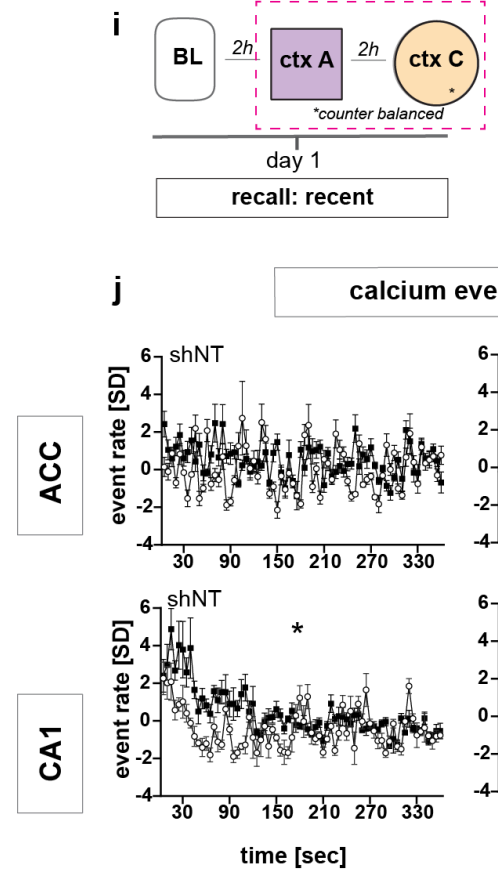

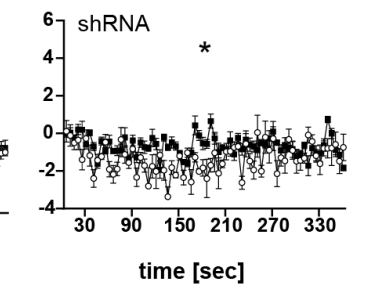

k
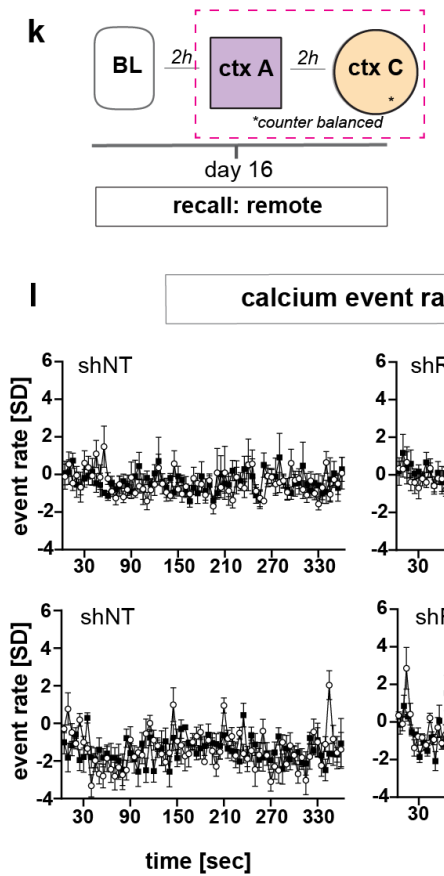
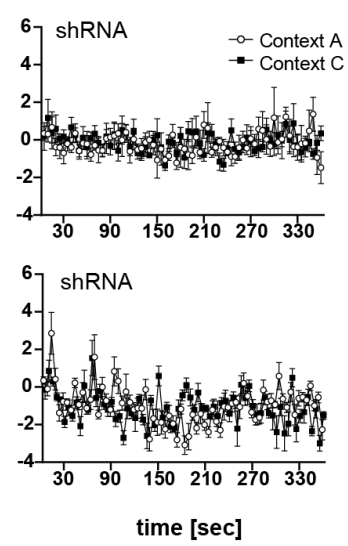


\section{$0 \quad$ Supplementary Figure 2: Supporting data for Figure 3}

1 a) Schematic of time point in behavioral paradigm of the presented data (context $\mathrm{A}$ and context $\mathrm{C}$ on day 1 after 2 foot-shock training in context A). b) Quantification of moving ratio as relative time in percentage of one minute 3 time bins. In both ACC and CA1, no difference was found between virus groups [Two-way repeated measures 4 ANOVA, ACC: virus $x$ time effect $p=0.554$, time main effect $p=0.08$, virus main effect $p=0.571$; CA1: virus $x$ 5 time effect $p=0.958$, time main effect $p=0.142$, virus main effect $p=0.856, n=5$ ]. c) Schematic of time point in 6 behavioral paradigm of the presented data (context $\mathrm{A}$ and context $\mathrm{C}$ on day 1 and day 16 after foot-shock training 7 in context A). d) Number of correlated pairs in percentage of all possible pairs (all neurons active in the context) 8 against the time mice were moving in the respective context. We found no correlation between the number of 9 correlated pairs and the time mice were moving. Pearson's $\mathrm{R}^{2}$ values are noted in brackets. e,g) Schematic of time 0 point in behavioral paradigm of the presented data (context $\mathrm{A}$ and context $\mathrm{C}$ on day 16 after foot-shock training 1 in context A).f,h) Quantification of $A \& C$ neurons that formed correlated pairs with other neurons (A\&C neurons 2 or context neurons) in ACC and CA1 (see also Figure $3 \mathrm{n}$ ) during recent (f) and remote (h) recall. In CA1, lenti3 shRNA injected mice showed significantly less A\&C neurons among the correlated pairs than shNT injected mice 4 [Unpaired t test with Welch's correction, $\mathrm{p}=0.017, \mathrm{n}=5$ ] at the remote time point. i) Schematic of time point in 5 behavioral paradigm of the presented data (context $\mathrm{A}$ and context $\mathrm{C}$ on day 1 after foot-shock training in context 6 A).j) Neuronal activity in form of normalized (to same day baseline) calcium event rate on day 1 after foot-shock 7 training (in context A) in both context $\mathrm{A}$ and context $\mathrm{C}$. All neurons active in the respective context were included 8 in this dataset. In CA1, the average event rate was higher in context $\mathrm{C}$ compared to context $\mathrm{A}$ in both virus groups 9 (left and right lower panel) [Two-way ANOVA with repeated measures, CA1, shNT: context $\mathrm{x}$ time effect $\mathrm{p}$ $0<0.0001$, time main effect $\mathrm{p}=0.007$, context main effect $\mathrm{p}=0.028, \mathrm{n}=5$, shRNA, : context $\mathrm{x}$ time effect $\mathrm{p}<0.0001$, 1 time main effect $p=0.012$, context main effect $p=0.029, n=5]$. No difference between contexts was found in ACC 2 [Two-way ANOVA with repeated measures, shNT: context $x$ time effect $p<0.0001$, time main effect $p=0.001$, 3 context main effect $p=0.216$, shRNA: context $x$ time $p<0.0001$, time main effect $p=0.156$, context main effect $4 \mathrm{p}=0.957 \mathrm{n}=5$ ]. k) Schematic of time point in behavioral paradigm of the presented data (context $\mathrm{A}$ and context $\mathrm{C}$ 5 on day 16 after foot-shock training in context A). l) Neuronal activity in form of normalized (to same day baseline) 6 calcium event rate on day 16 after foot-shock training (in context $\mathrm{A}$ ) in both context $\mathrm{A}$ and context $\mathrm{C}$. All neurons 7 active in the respective context were included in this dataset. There was no context effect in any experimental 8 group [Two-way ANOVA with repeated measures, ACC, shNT: context $x$ time effect $p=0.456$, time main effect $9 \mathrm{p}=0.321$, context main effect $\mathrm{p}=0.839$; shRNA: context $\mathrm{x}$ time effect $\mathrm{p}=0.863$, time main effect $\mathrm{p}=0.446$, context 0 main effect $\mathrm{p}=0.73, \mathrm{n}=5$; CA1, shNT: context $\mathrm{x}$ time effect $\mathrm{p}<0.0001$, time main effect $\mathrm{p}=0.0004$, context main 1 effect $\mathrm{p}=0.953$, shRNA: context $\mathrm{x}$ time effect $\mathrm{p}<0.0001$, time main effect $\mathrm{p}=0.005$, context main effect $\mathrm{p}=0.603$, $2 \mathrm{n}=5$ ]. 
bioRxiv preprint doi: https://doi.org/10.1101/2021.05.21.445117; this version posted May 21, 2021. The copyright holder for this preprint (which

was not certified by peer review) is the author/funder, who has granted bioRxiv a license to display the preprint in perpetuity. It is made available under aCC-BY-NC-ND 4.0 International license.

a

Observed data - - Same cell model - - - threshold
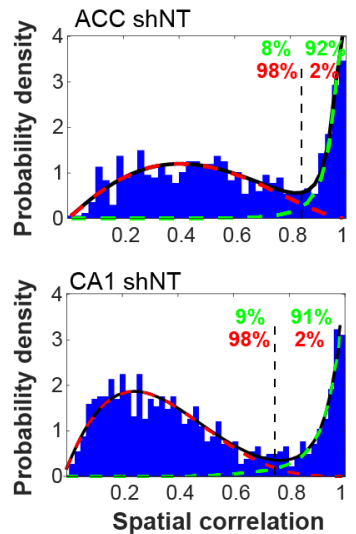

b

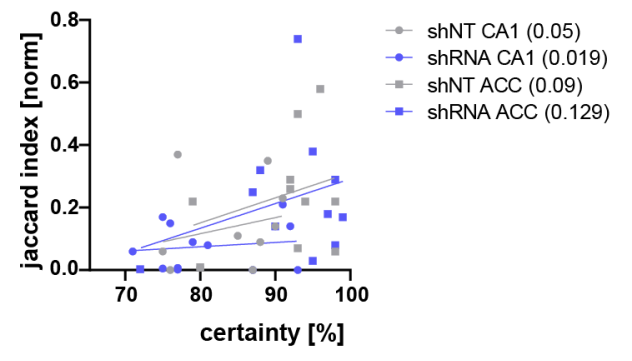

suppl Figure 3

C
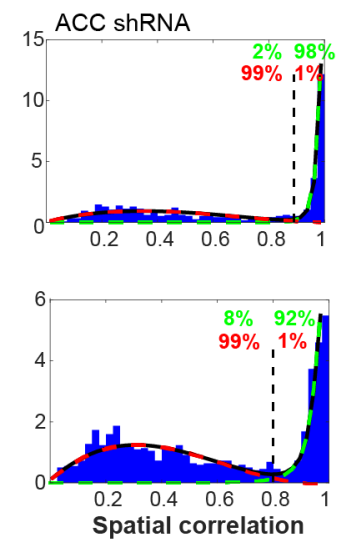

U
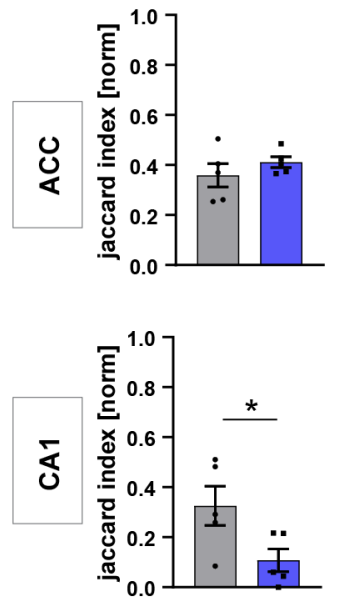

\section{Quantification of A\&C neurons}

day 1

day 16
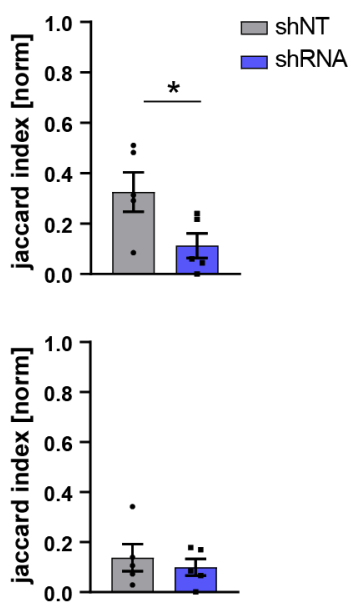


\section{$4 \quad$ Supplementary Figure 3: Supporting data for Figure 3}

5 a) Example distributions of spatial correlation generated with CellReg (Sheintuch et al., 2017) on spatial maps 6 from each experimental group using probabilistic modeling. The green and red numbers indicate the certainty of 7 each model (same cell model, green; different cell model, red). All groups showed a bimodal distribution though 8 the distributions and thresholds differed between ACC (upper panels) and CA1 (lower panels) but not between 9 treatment groups within each brain region (left and right panels). b) Plot of registration certainty (green numbers 0 in example a) against the normalized number of $\mathrm{A} \& \mathrm{C}$ neurons (normalized jaccard similarity index) on day 1 and 1 day 16. There was no significant correlation between the certainty of cell registration and the number of A\&C 2 neurons (Pearson's $\mathrm{R}^{2}$ is noted in brackets). c) Quantification of A\&C neurons using a fixed threshold for spatial 3 correlation of 0.85 . The effects found in Figure 3 were similar to those found using a fixed threshold [Unpaired $t$ 4 test with Welch's correction, day 1, ACC: $\mathrm{p}=0.35$, CA1 p=0.05; day 16, ACC p=0.044, CA1 p=0.567; n=5]. 
bioRxiv preprint doi: https://doi.org/10.1101/2021.05.21.445117; this version posted May 21, 2021. The copyright holder for this preprint (which

was not certified by peer review) is the author/funder, who has granted bioRxiv a license to display the preprint in perpetuity. It is made available under aCC-BY-NC-ND 4.0 International license.
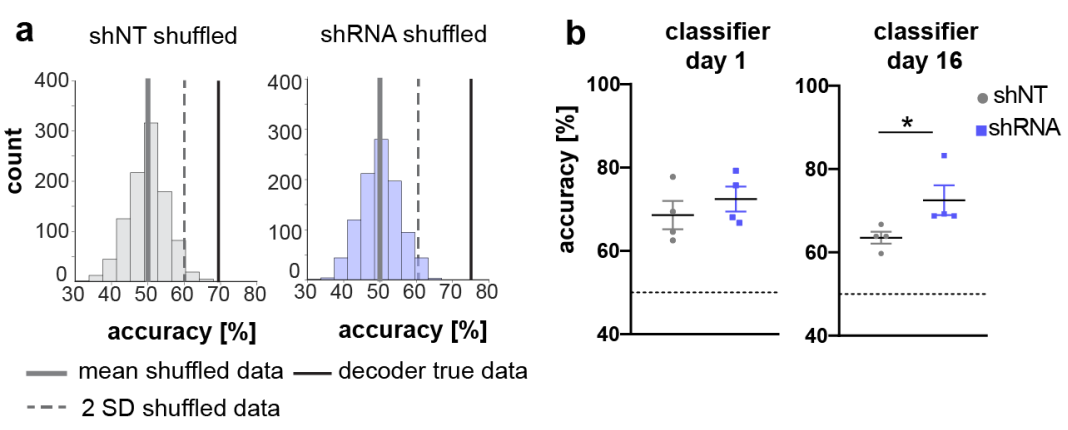

C

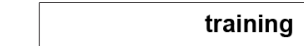

training recent recall remote recall

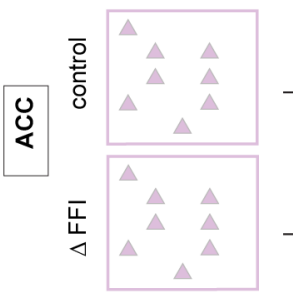
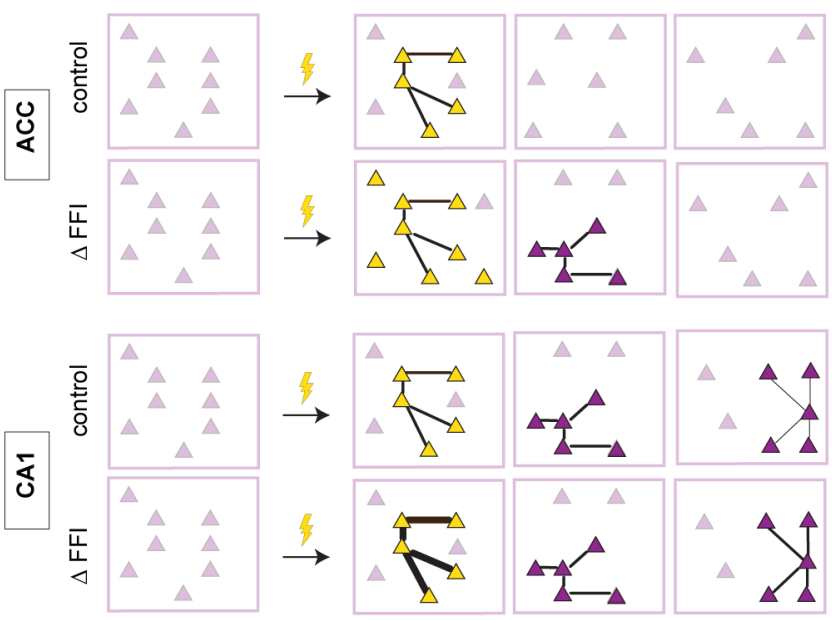

$\triangle$ active neuron $\triangle$ footshock responsive neuron

$\Delta$ active, high correlated neuron

correlation strength

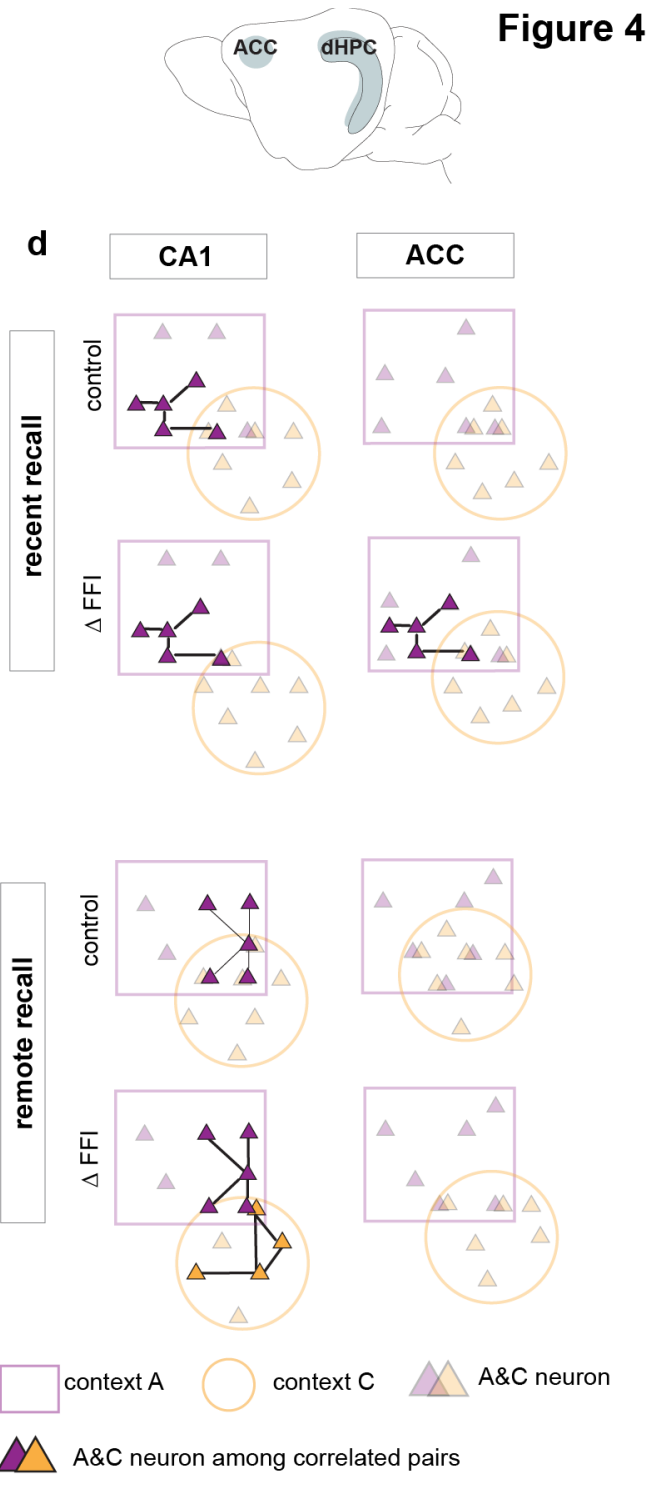

Figure 4 
Figure 4: Increased FFI in DG-CA3 facilitates evolution of context-specific neuronal ensembles at remote timepoint

a) A support vector machine was trained to classify the context based on neuronal activity of CA1 A\&C neurons.

1 A distribution of prediction accuracy was created with 1000 randomized datasets of shuffled event times 8 (shuffled). Graphs show an example distribution of an shNT-injected (left) and an shRNA-injected mouse (right) 9 recent recall (day 1). Lines in graph indicate the mean (solid) and 2 standard deviations from the mean (dashed). 0 The solid line indicates the decoder accuracy of the true data from the respective mouse. b) Summary of prediction 1 accuracy (within subject) for day 1 and day 16. On day 1, the accuracy to predict the context based on A\&C 2 neuron's activity was above chance (also see shuffled data in panel a) [One-sample t test against 50 (chance level), $3 \operatorname{shNT}, \mathrm{p}=0.012$, shRNA, $\mathrm{p}=0.005, \mathrm{n}=4$ (only mice with $>50$ A\&C neurons were included to avoid overfitting) 4 with no difference between the treatment groups [Mann Whitney test, $\mathrm{p}=0.486, \mathrm{n}=4$ ]. This was maintained on 5 day 16 [One-sample t test against 50 (chance level), shNT, p =0.003, shRNA, p =0.008, $\mathrm{n}=4$ ] with a significantly 6 higher accuracy in lenti-shRNA injected mice [Mann Whitney test, $p=0.029, n=4$ ]. c-d) Graphic summary of 7 presented findings. Learning induced the formation of neuronal ensembles of foot-shock-responsive neurons in 8 both ACC and CA1 and FFI potentiated this property in CA1. During early stages of memory consolidation 9 (recall of recent memory), we observed the emergence of training context-associated ensembles (increased 0 numbers of co-active neurons) in both CA1 and ACC. Increasing FFI in DG-CA3 promoted emergence of training 1 context-associated ensemble in ACC suggestive of increased CA1-ACC communication (c). Additionally, we 2 observed a FFI dependent reduction in the number of neurons active in both training and neutral contexts in 3 CA1(d, top) reflecting increased specificity of neuronal ensembles. At remote recall, we observed that FFI in 4 DG-CA3 prevented time-dependent decay of the training context-associated ensemble and promoted acquisition 5 (or maintenance since it may have emerged prior to test at remote timepoint) of a neutral context specific ensemble 6 in CA1 (c, d). Within the ACC, and not evident at the recent timepoint, we observed a FFI dependent reduction 7 in the number of neurons active in both training and neutral contexts (d, bottom). 